The Astrophysical Journal, 505:390-417, 1998 September 20

(C) 1998. The American Astronomical Society. All rights reserved. Printed in U.S.A.

\title{
HELIOSEISMIC STUDIES OF DIFFERENTIAL ROTATION IN THE SOLAR ENVELOPE BY THE SOLAR OSCILLATIONS INVESTIGATION USING THE MICHELSON DOPPLER IMAGER
}

\author{
J. Schou, ${ }^{1}$ H. M. ANtia, ${ }^{2}$ S. BAsu, ${ }^{3,14}$ R. S. Bogart, ${ }^{1}$ R. I. Bush, ${ }^{1}$ S. M. Chitre, ${ }^{2}$ J. Christensen-DalsgaARD, ${ }^{3,4}$ \\ M. P. Di Mauro, ${ }^{11}$ W. A. Dziembowski,${ }^{12}$ A. EFf-Darwich, ${ }^{5}$ D. O. Gough, ${ }^{8,13}$ D. A. Haber, ${ }^{7}$ \\ J. T. HoEKSEMA, ${ }^{1}$ R. Howe, ${ }^{6,15}$ S. G. KorzenNiK, ${ }^{5}$ A. G. KosoviChev,${ }^{1}$ R. M. LARSEN $,{ }^{7}, 10$ \\ F. P. PiJPers, ${ }^{3}$ P. H. Scherrer, ${ }^{1}$ T. SeKiI, ${ }^{8}$ T. D. TARbell, ${ }^{9}$ A. M. Title,${ }^{9}$ \\ M. J. ThOMPSON, ${ }^{6}$ AND J. TOOMRE ${ }^{7}$ \\ Received 1997 December 2; accepted 1998 April 29
}

\begin{abstract}
The splitting of the frequencies of the global resonant acoustic modes of the Sun by large-scale flows and rotation permits study of the variation of angular velocity $\Omega$ with both radius and latitude within the turbulent convection zone and the deeper radiative interior. The nearly uninterrupted Doppler imaging observations, provided by the Solar Oscillations Investigation (SOI) using the Michelson Doppler Imager (MDI) on the Solar and Heliospheric Observatory (SOHO) spacecraft positioned at the $L_{1}$ Lagrangian point in continuous sunlight, yield oscillation power spectra with very high signal-tonoise ratios that allow frequency splittings to be determined with exceptional accuracy. This paper reports on joint helioseismic analyses of solar rotation in the convection zone and in the outer part of the radiative core. Inversions have been obtained for a medium- $l$ mode set (involving modes of angular degree $l$ extending to about 250) obtained from the first 144 day interval of SOI-MDI observations in 1996. Drawing inferences about the solar internal rotation from the splitting data is a subtle process. By applying more than one inversion technique to the data, we get some indication of what are the more robust and less robust features of our inversion solutions. Here we have used seven different inversion methods. To test the reliability and sensitivity of these methods, we have performed a set of controlled experiments utilizing artificial data. This gives us some confidence in the inferences we can draw from the real solar data. The inversions of SOI-MDI data have confirmed that the decrease of $\Omega$ with latitude seen at the surface extends with little radial variation through much of the convection zone, at the base of which is an adjustment layer, called the tachocline, leading to nearly uniform rotation deeper in the radiative interior. A prominent rotational shearing layer in which $\Omega$ increases just below the surface is discernible at low to mid latitudes. Using the new data, we have also been able to study the solar rotation closer to the poles than has been achieved in previous investigations. The data have revealed that the angular velocity is distinctly lower at high latitudes than the values previously extrapolated from measurements at lower latitudes based on surface Doppler observations and helioseismology. Furthermore, we have found some evidence near latitudes of $75^{\circ}$ of a submerged polar jet which is rotating more rapidly than its immediate surroundings. Superposed on the relatively smooth latitudinal variation in $\Omega$ are alternating zonal bands of slightly faster and slower rotation, each extending some $10^{\circ}$ to $15^{\circ}$ in latitude. These relatively weak banded flows have been followed by inversion to a depth of about $5 \%$ of the solar radius and appear to coincide with the evolving pattern of "torsional oscillations" reported from earlier surface Doppler studies.
\end{abstract}

Subject headings: convection - Sun: interior - Sun: oscillations - Sun: rotation

\section{INTRODUCTION}

The Sun, like all moderate-mass main-sequence stars, has an extensive convective envelope. Energy is efficiently transported by fluid motion, except in a thin superadiabatic boundary layer immediately beneath the photosphere in which there is a transition to transport by radiation. There is possibly also a similar transition layer at the bottom of the zone. In the deeper interior, radiation is able to carry the energy from the nuclear-burning core, and indeed in the so-called standard theories of solar evolution it is assumed to do so. Nevertheless, dynamical transport is certainly not

\footnotetext{
${ }^{1}$ W. W. Hansen Experimental Physics Laboratory, Stanford University, Stanford, CA 94305-4085.

${ }^{2}$ Tata Institute of Fundamental Research, Mumbai 400005, India.

3 Teoretisk Astrofysik Center, Danmarks Grundforskningfond, Denmark.

${ }^{4}$ Institut for Fysik og Astronomi, Aarhus Universitet, DK-8000 Aarhus C, Denmark.

${ }^{5}$ Harvard-Smithsonian Center for Astrophysics, 60 Garden Street, Cambridge, MA 02138.

${ }^{6}$ Astronomy Unit, Queen Mary and Westfield College, University of London, E1 4NS, UK.

7 JILA and Department of Astrophysical and Planetary Sciences, University of Colorado, Boulder, CO 80309-0440.

${ }^{8}$ Institute of Astronomy, University of Cambridge, Madingley Road, Cambridge, CB3 0HA, UK.

${ }^{9}$ Lockheed Martin Advanced Technology Center, Org. H1-11, Building 252, 3251 Hanover Street, Palo Alto, CA 94304.

${ }^{10}$ Datalogisk Institut, Aarhus Universitet, DK-8000 Aarhus C, Denmark.

${ }_{11}^{1}$ CNR-Gruppo Nazionale di Astronomia, UdR di Catania Istituto di Astronomia, Universita' di Catania, Viale A. Doria 6, I-95125 Catania, Italy.

12 Copernicus Astronomical Center, ul. Bartycka 18, 03-610 Warszawa, Poland.

${ }^{13}$ Department of Applied Mathematics and Theoretical Physics, University of Cambridge, Silver Street, Cambridge, CB3 9EW, UK.

14 Present address: School of Natural Sciences, Institute for Advanced Study, Princeton, NJ 08540.

${ }^{15}$ Present address: National Solar Observatory, National Optical Astronomy Observatories, P.O. Box 26732, Tucson, AZ 85726-6732.
} 
precluded. In the convective envelope, which in the Sun occupies the outer $30 \%$ by radius, intense turbulence not only transports heat but also continually redistributes angular momentum. That leads to a pronounced nonuniformity in the angular velocity. Observation of features such as sunspots on the Sun's surface, augmented subsequently by direct Doppler measurements, made it apparent long ago that the Sun rotates differentially: there appears to be a smooth poleward decline in the angular velocity of the surface layers, the sidereal rotation period being about 25 days in equatorial regions and more than a month near the poles. The convection also appears to build and rebuild magnetic fields by some form of magnetohydrodynamic action, the details of which are thought to depend sensitively on the rotation profile that is established both within the convection zone and in a region of overshooting immediately beneath its base (e.g., Gilman 1986; Solanki 1993; Cattaneo 1993; Wilson 1994). However, just how the turbulence couples to the rotation and the magnetic field is not understood; it is extremely difficult to describe theoretically the dynamics of such highly nonlinear compressible fluid flow over many decades in scale (cf. Brummell, Cattaneo, \& Toomre 1995). Yet one of the striking aspects of such dynamics is that in the midst of apparent chaos there appears also to be some order, as is suggested partly by the relatively smooth variation of angular velocity observed at the solar surface and by the nearly cyclic temporal variation of magnetic activity, in which both the polarity of the field and the latitudes at which sunspots emerge follow welldefined rules.

The nature of the rotation profile achieved in a Sun-like star touches many issues beyond the dynamics of the convection zone. Of particular interest is the manner in which an evolved star may have been spun down to its present state of relatively slow surface rotation. The gradual loss of angular momentum over the main-sequence life of the Sun was probably accomplished by magnetic coupling to the solar wind (e.g., Rosner \& Weiss 1985; Mestel \& Weiss 1987; Charbonneau \& MacGregor 1993), spinning down the convective envelope and probably much of the deeper interior. Associated with most rotational decelerations of fluid systems are slow meridional circulations, which in the Sun can gradually redistribute the chemical composition, thereby playing a central role in the permanent depletion of lighter elements such as lithium from the photosphere. However, the radial extent of spin-down is difficult to assess theoretically, owing to our inability to assess reliably the possibly delicate balance between transport by laminar circulation, instability, mechanical waves, and magnetic fields, which can either enhance or suppress rotational shear (e.g., Gough 1977; Spruit 1987; Hartmann \& Noyes 1987; Chaboyer, Demarque, \& Pinsonneault 1995; Kumar \& Quataert 1997; Zahn, Talon, \& Matias 1997; Gough 1997). Such uncertainty admits the possibility that the central region of the Sun may still retain some vestige of its primordial past, such as a rapidly rotating core which may even be highly magnetized. The apparent deficit in the neutrinos emanating from the nuclear-burning core has encouraged dynamical studies of readjustment of chemical composition and thermal stratification, associated with which is redistribution of angular momentum (e.g., Gough 1988; Ghosal \& Spiegel 1991; Merryfield, Toomre, \& Gough 1991).

Numerical simulations of convection in rotating spherical shells (e.g., Glatzmaier 1985, 1987; Gilman \& Miller
1986) suggest that the flow in the solar convection zone is dominated by columnar roll-like cells (or "banana cells") oriented approximately in the north-south direction, but with a slight tilt, causing the Reynolds stresses to drive zonal flows, which are manifest as differential rotation. The models have yielded angular velocities $\Omega$ which are nearly constant along the axes of the cells and thus nearly constant on cylinders aligned with the axis of rotation, the magnitude of $\Omega$ decreasing with depth at essentially all latitudes.

In the past $20 \mathrm{yr}$ helioseismology has made it possible to begin to probe the variation with depth and latitude of both sound speed and angular velocity in the Sun (e.g., Gough \& Toomre 1991; Harvey 1995) and thereby test the theoretical ideas. This has led to some major revelations. In particular, inversion of p-mode frequency splitting (e.g., Duvall et al. 1984; Christensen-Dalsgaard \& Schou 1988; Libbrecht 1989; Brown et al. 1989; Dziembowski, Goode, \& Libbrecht 1989; Gough et al. 1993; Tomczyk, Schou, \& Thompson 1995a; Thompson et al. 1996 and review therein) has shown that the variation of angular velocity in the convection zone is quite different from earlier theoretical predictions based on global simulations. Although there is some variation with radius, as we discuss later in this paper, the overall picture is that there is little radial variation in the convection zone: the latitudinal variation of $\Omega$ observed in the surface layers extends through much of the convective envelope (e.g., Thompson et al. 1996). Therefore, much of the theoretical intuition about differential rotation in the convection zone that has been garnered from the simulations is thrown into doubt.

There are other theoretical approaches based on modified mixing-length and anisotropic-diffusion models, which have been used to estimate the turbulent Reynolds stresses which drive the global-scale flows (e.g., Gough 1978; Rüdiger 1980, 1989; Hathaway 1984; Durney 1985, 1991), and these have resulted in a variety of other rotation profiles. Unfortunately, there has been no substantial independent experimental test of these prescriptions, so it is unclear which, if any, should be preferred. Understanding the solar rotation may require simulations of convection in rotating spherical shells that are capable of attaining fully developed turbulence in low Prandtl number compressible fluids at very high Reynolds numbers. Only under such conditions might turbulence redistribute angular momentum on a large scale in a manner that is similar to what we observe on the Sun, and that may be quite different from the almost laminar or mildly turbulent convection that was studied in the early simulations (e.g., Brummell, Hurlburt, \& Toomre 1996, 1998).

The helioseismological studies have revealed also that immediately beneath the base of the convection zone there is a region of strong shear, known as the tachocline, whose role has been discussed by, for example, Gilman, Morrow, \& DeLuca (1989) and Spiegel \& Zahn (1992). This is a region of transition between the latitudinally varying rotation of the convection zone and the nearly uniform rotation that is observed deeper in the radiative interior. (We report in this paper that the shear extends somewhat into the convection zone, particularly at high latitudes.) There is also a thin shear layer near the solar surface, in which $\Omega$ increases with depth at intermediate and low latitudes. The cause of this shear is unexplained.

In this paper, we report on the latest inferences concerning solar differential rotation that have been drawn from the 
helioseismic data that are now available from the Solar Oscillations Investigation (SOI) using the Michelson Doppler Imager (MDI) on the Solar and Heliospheric Observatory (SOHO). That spacecraft is positioned in a halo orbit near the Sun-Earth Lagrangian point $L_{1}$ in order to obtain continuous Doppler-imaged observations of the Sun with high spatial fidelity. The MDI instrument has a pair of tunable Michelson interferometers through which the Sun is imaged onto a $1024^{2}$ charge-coupled device camera. Doppler velocity, intensity, and magnetic field images are recorded, based on modulations of the $676.8 \mathrm{~nm} \mathrm{Ni}$ I solar absorption line. The data span either the whole solar disk or a magnified portion of it (e.g., Scherrer et al. 1995). The high spatial resolution of MDI thereby permits the study of many millions of global resonant modes of solar oscillation. These space-based observations complement the groundbased observations from instruments such as the Global Oscillation Network Group (GONG) (Harvey et al. 1996). Determination and subsequent inversion of the frequencies of these modes, including the degeneracy splitting, enable us to infer how the angular velocity varies throughout much of the interior of the Sun.

The solution of the inverse problem is inherently nonunique. We have only a finite number of data, and we are trying to infer the angular velocity as a function of position in the solar interior. If the aim is to find as a solution a function $\Omega(r, \theta)$ that fits the data, then mathematically there must be infinitely many functions that fit the data equally well. Another problem is that the inverse problem is ill conditioned, so that the simplest least-squares fit to the data gives a solution that is dominated by the noise in the data. To solve this second problem we have to regularize the inversion solutions in some way. One such approach is the regularized least-squares method. This also "solves" the problem of nonuniqueness, since having decided on a particular form of regularization and other details of the implementation, the inversion method comes up with a single solution to the regularized problem. Other choices of regularization will in general lead to different solutions, however, and there is no way mathematically or physically to choose infallibly between the different solutions. (The optimally localized averages methods - see $\S 3$-are based on a different philosophy. They do not set out to produce a global solution that fits the data but instead seek localized averages which can be interpreted as a blurred representation of the true underlying rotation profile. The blurring is made precise in terms of averaging kernels.) Because different methods come up with different solutions to the nonunique inverse problem, we consider it instructive to compare results from a number of different methods. We can see which features appear to be robust, in as much as they are common to many methods, and the differences in the solutions give some indication of the uncertainty in the inferences. More precise understanding of the common features and differences between the methods can be obtained by using the averaging kernels and by inverting artificial data. Thus, in order to test the sensitivity and reliability of the inversion techniques used in this study, and hence to assess the reliability of our inferences about the Sun, we have first applied the methods in a blind experiment (socalled hare and hounds) to artificial data computed from fictitious (but not wholly unrealistic) rotation profiles. The mode set and splitting coefficients were selected to match the range of SOI-MDI data available, and noise similar in magnitude to the estimated errors in the data was added to the artificial splittings. This controlled experiment gives us increased confidence in the solar results we then present.

Illustrating the results of many inversion methods economically presents its own problems. In some figures we have used contour plots showing one quadrant of the solar interior (the rest of the interior can be obtained by reflection in the equatorial plane and rotation about the polar axis, since the inversion solutions, their errors, and their averaging kernels all share these symmetries.) In such plots we have chosen to use just four of the inversion methods, since to illustrate all the methods would have doubled the space required. The four methods used were chosen simply to illustrate the broad diversity of approaches. In other figures it has been instructive to take cuts through the inversion solutions at fixed radius or at fixed latitude. This representation has the added benefit that we have been able to include results from all the inversion methods.

Because of the intrinsic nonuniqueness of all inversion methods, combined with various trade-offs within each method between spatial resolution and error amplification, we are not in a position to advocate which method and resulting solution is the "best" one. However, we shall comment on which inferences concerning the angular velocity $\Omega$ appear to be robust and which ones are somewhat less certain.

The layout of the rest of the paper then is as follows. We discuss the observations and the data set in $\S 2$. In $\S 3$ we present details of the inversion techniques, and in $\S 4$ we summarize the procedure and results of the hare-andhounds experiment. Solar results are presented in $\S 5$, followed in $\S 6$ by a discussion of their physical implications.

\section{SOI-MDI ROTATIONAL SPLITTING DATA}

The Sun oscillates simultaneously in many millions of modes. Helioseismology uses the observed properties of these modes to make inferences about the Sun's internal structure and dynamics. Each mode can be identified by three integers $(n, l, m)$, where $l$ and $m$ are the degree and order respectively of a spherical harmonic and $n$ is the radial order of the mode, which is for most modes the number of radial nodes in the vertical displacement eigenfunction. The azimuthal order $m$ can take all values from $-l$ to $+l$. Each mode has a sinusoidal time dependence with angular frequency $\omega_{n l m}$ (or equivalently, cyclic frequency $\left.v_{n l m} \equiv \omega_{n l m} / 2 \pi\right)$. In a spherically symmetric, nonrotating star, the frequency $\omega_{n l m}$ of an eigenmode would be independent of $m$ and thus there would be multiplets of $(2 l+1)$ modes with identical frequencies $\omega_{n l}$, each multiplet corresponding to an $(n, l)$ pair. Departures from spherical symmetry lift this $(2 l+1)$-fold degeneracy, inducing a splitting $\omega_{n l m}-\omega_{n l}$ in frequency between modes in the same multiplet. In particular, to leading order the rotational splitting is determined by the angular velocity $\Omega(r, \theta)$ inside the Sun:

$$
\Delta \omega_{n l m} \equiv \omega_{n l m}-\omega_{n l}=\int_{0}^{R} \int_{0}^{\pi} K_{n l m}(r, \theta) \Omega(r, \theta) r d r d \theta,
$$

where $r$ is distance to the center, $\theta$ is the colatitude, and $R$ is the total radius of the Sun (e.g., Schou, ChristensenDalsgaard, \& Thompson 1994). The functions $K_{n l m}(r, \theta)$, which we shall refer to as the mode kernels, are functions of the mean spherically symmetric structure of the Sun. The kernels are symmetric in $\theta$ around the equator; thus it 
follows from equation (1) that the splittings are sensitive only to the similarly symmetric component of $\Omega(r, \theta)$. We also note that the kernels, and hence the splittings, are odd functions of $m$, since they depend on the direction of propagation of the modes in longitude, relative to the rotation. (In contrast, departures from spherical symmetry in solar structure cause frequency shifts that are even functions of $m$.)

The dependence of the splittings on the internal rotation rate can be used in an inverse problem to probe the Sun's internal rotation. In particular, it allows one to perform two-dimensional inversions for the rotation rate as a function of radius and latitude. We assume that the mode kernels are known and $\Omega$ is the unknown function that we seek to infer. The data are frequency splittings (or more precisely, splitting coefficients that are combinations of the mode splittings; see below). Inevitably the data also contain noise.

In this paper, we have taken frequency splittings determined from the observations of SOI-MDI and applied various inversion techniques for estimating from the data the internal rotation of the solar interior. The high duty cycle (over 95\%) of these observations and the lack of atmospheric seeing variations make this data set superb for our purpose. Also we have been able to use splitting coefficients (see below) up to index $j=36$, which has allowed us to obtain unprecedented latitudinal resolution and to push the reliable region of our inversions closer to the poles.

The data come from the medium-l program of SOI-MDI. This program, which is described more fully by Kosovichev et al. (1997), is designed to be sensitive to solar p modes up to roughly $l=300$. The data are spatial averages of Doppler velocity over the solar disk, in $10^{\prime \prime}$ bins. After appropriate remapping and projection onto eigenmode masks, the time series of projected images are Fourier transformed in time to produce power spectra corresponding to different $(l, m)$ pairs. Modes of different $n$ give rise to different peaks in the spectra. The frequencies of the normal modes must be estimated from the location of the peaks. This is achieved by fitting a model of the peak structure to the power spectra or directly to the Fourier transforms. Details of the method for frequency estimation were given by Schou (1992); see also Hill et al. (1996) for another discussion of the data analysis issues. In order to increase the stability of the fit, we do not attempt to determine the frequencies $\omega_{n l m}$ of individual modes. Instead we parameterize the $2 l+1$ frequencies within a given $(n, l)$ multiplet as

$$
\omega_{n l m} / 2 \pi=v_{n l}+\sum_{j=1}^{j_{\max }} a_{j}(n, l) \mathscr{P}_{j}^{(l)}(m),
$$

with generally fewer than $2 l+1$ parameters $v_{n l}, a_{1}, a_{2}, \ldots$, $a_{j_{\max }}$, and fit this expression to the peaks in the Fourier spectra. The basis functions $\mathscr{P}_{j}^{(l)}(m)$ used in this expansion are polynomials of degree $j$ defined by

$$
\mathscr{P}_{j}^{(l)}(l)=l, \quad \text { and } \quad \sum_{m=-l}^{l} \mathscr{P}_{i}^{(l)}(m) \mathscr{P}_{j}^{(l)}(m)=0 \quad \text { for } i \neq j .
$$

They are related to Clebsch-Gordan coefficients $C_{j 0 l m}^{l m}$ by

$$
\mathscr{P}_{j}^{(l)}(m)=\frac{l \sqrt{(2 l-j) !(2 l+j+1) !}}{(2 l) ! \sqrt{2 l+1}} C_{j 0 l m}^{l m}
$$

[e.g., Edmonds 1959; see also Pijpers 1997 for explicit expressions for the $\left.\mathscr{P}_{j}^{(l)}\right]$. We note that it follows from the symmetry properties of the splittings that rotation contributes only to the $a_{j}$ for odd $j$.

It will be useful for the development of the inversion methods and the interpretation of their results to point out at this stage that the splitting coefficients are just linear combinations of the frequency splittings:

$$
2 \pi a_{j}(n, l)=\sum_{m} \gamma_{j}(l, m) \Delta \omega_{n l m},
$$

(e.g., Schou et al. 1994) for some coefficients $\gamma_{j}(l, m)$. It therefore follows from equation (1) that the odd splitting coefficients are weighted averages of the angular velocity $\Omega(r, \theta)$ :

$$
\begin{aligned}
2 \pi a_{2 s+1}(n, l) & =\int_{0}^{R} \int_{0}^{\pi} \sum_{m} \gamma_{2 s+1}(l, m) K_{n l m}(r, \theta) \Omega(r, \theta) r d r d \theta, \\
& \equiv \int_{0}^{R} \int_{0}^{\pi} K_{n l s}^{(a)} \Omega r d r d \theta .
\end{aligned}
$$

Thus, whether the data are taken to be frequency splittings or (as in our case) splitting coefficients, they are linear functionals of the angular velocity in the solar interior.

The data used for this work were obtained from 144 days of SOI-MDI medium-l observations made between 1996 May 9 and September 29. The (non-gap-filled) time series had a duty cycle of $95.47 \%$. Fits were made with $j_{\max }=6$, $j_{\max }=18$, and $j_{\max }=36$. For each multiplet one of these fits was chosen depending on the value of $l$ and the convergence of the fits. The combined set is made up of a total of 30,648 splitting coefficients $a_{j}$ (for odd $j$, up to $a_{35}$ ) from $2036(n, l)$ multiplets. The degree $l$ ranges from 1 to 250 , and the multiplet frequency $v_{n l}$ from 954 to $4556 \mu \mathrm{Hz}$. The f-mode (i.e., $n=0$ ) splitting coefficients are included in the data set for degrees $l=88-250$; but for $\mathrm{p}$ modes the highest degree present is $l=194$ (for $n=1$ ). The extent of the set of multiplets for which data are available is governed by how reliably the mode parameters can be determined in the fitting. At high degrees and high frequencies the fitting becomes unreliable as the peaks in the power spectrum become too closely spaced relative to their width (e.g., Howe \& Thompson 1998a, 1998b). The standard deviations of the probable errors in the splittings were determined internally by the fitting algorithm. In the middle of our $l$-range (say, around $l=80$ ) and at a frequency of about $3 \mathrm{mHz}$, the error in $a_{j}$ is about $0.2-0.3 \mathrm{nHz}$ for $j=1$ (compared with the value of $a_{1}$ itself of about $440 \mathrm{nHz}$ ), increases with index $j$ to about $0.4-0.5 \mathrm{nHz}$ for $j \simeq 9$, and then drops off to $0.02-0.03 \mathrm{nHz}$ for $j=35$ (which is the same order of magnitude as $a_{35}$ itself).

\section{INVERSION TECHNIQUES}

The data $d_{i}$, which are either splittings or splitting coefficients, are weighted averages of the Sun's internal angular velocity $\Omega$ (cf. eqs. [1] and [6]):

$d_{i}=\int_{0}^{\pi / 2} \int_{0}^{R} K_{i}(r, \theta) \Omega(r, \theta) r d r d \theta+\epsilon_{i}, \quad i=1, \ldots, M ;$

here $i$ labels the data, the $K_{i}$ are the corresponding kernels, and $\epsilon_{i}$ are the errors, with standard deviations $\sigma_{i}$. In the present case, the errors are essentially uncorrelated, and in the following we shall assume that this is strictly true.

To use these data to make inferences about the rotation, based on equation (7), is a problem in inverse theory. We have used seven inversion methods. We briefly outline these 
methods in this section; further details will be found in the literature referenced.

The techniques discussed here are all linear, in the sense that the inferred solution $\bar{\Omega}\left(r_{0}, \theta_{0}\right)$ results as a linear combination of the data; from equation (7) it therefore follows that

$$
\begin{aligned}
\bar{\Omega}\left(r_{0}, \theta_{0}\right) \equiv & \sum_{i=1}^{M} c_{i}\left(r_{0}, \theta_{0}\right) d_{i} \\
= & \sum_{i=1}^{M} c_{i}\left(r_{0}, \theta_{0}\right) \int_{0}^{\pi / 2} \int_{0}^{R} K_{i}(r, \theta) \Omega(r, \theta) r d r d \theta \\
& +\sum_{i=1}^{M} c_{i}\left(r_{0}, \theta_{0}\right) \epsilon_{i} \\
= & \int_{0}^{\pi / 2} \int_{0}^{R} \mathscr{K}\left(r_{0}, \theta_{0}, r, \theta\right) \Omega(r, \theta) r d r d \theta \\
& +\sum_{i=1}^{M} c_{i}\left(r_{0}, \theta_{0}\right) \epsilon_{i},
\end{aligned}
$$

where

$$
\mathscr{K}\left(r_{0}, \theta_{0}, r, \theta\right)=\sum_{i=1}^{M} c_{i}\left(r_{0}, \theta_{0}\right) K_{i}(r, \theta),
$$

for suitable inversion coefficients $c_{i}\left(r_{0}, \theta_{0}\right)$. Since the inversion methods in general recover precisely a constant angular velocity from error-free artificial data it follows that $\mathscr{K}$ is normally unimodular and that the solution can thus be regarded as an average of the true $\Omega(r, \theta)$, weighted by the averaging kernel $\mathscr{K}$ and with variance

$$
\sigma\left[\bar{\Omega}\left(r_{0}, \theta_{0}\right)\right]^{2}=\sum_{i=1}^{M}\left[c_{i}\left(r_{0}, \theta_{0}\right) \sigma_{i}\right]^{2} .
$$

Some of the techniques discussed below proceed by determining the inversion coefficients directly, while in other cases these would have to be derived by a separate, sometimes cumbersome, calculation.

The kernels $K_{n l m}$ for the splittings $\Delta \omega_{n l m}$ are almost separable, since they can be written as

$$
K_{n l m}=F_{1 n l}(r) G_{1 l m}(\theta)+F_{2 n l}(r) G_{2 l m}(\theta),
$$

where in general the second term is considerably smaller, by a factor of order $l^{2}$, than the first. The kernels $K_{n l s}^{(a)}$ for $a$ coefficients can be separated similarly (cf. Pijpers 1997). This form of the kernels can be utilized in developing efficient inversion algorithms.

The expansion of the splittings in polynomials in $m$, as in equation (2), corresponds to an expansion of $\Omega$ as

$$
\Omega(r, \theta)=\sum_{s=0}^{s_{\max }} \Omega_{s}(r) \psi_{2 s}^{(1)}(x),
$$

in polynomials $\psi_{2 s}^{(1)}(x)$ in $x \equiv \cos \theta$ (e.g., Brown et al. 1989). If the $\psi_{2 s}$ are chosen as

$$
\psi_{2 s}^{(1)}(x)=\frac{d P_{2 s+1}}{d x},
$$

$\left[P_{k}(x)\right.$ being a Legendre polynomial], the $a$-coefficients and expansion functions for $\Omega$ are related by

$$
2 \pi a_{2 j+1}(n, l)=\int_{0}^{R} K_{n l j}^{j}(r) \Omega_{j}(r) d r,
$$

for suitable kernels $K_{n l j}^{j}$ (Ritzwoller \& Lavely 1991; see also Pijpers 1997). Thus the original inverse problem has been decomposed into a series of independent inversions in $r$; this forms the basis for the so-called 1.5-dimensional inversion methods.

All inversion methods depend on parameters that control the trade-off between the error in the solution and the resolution, possibly allowing a separate trade-off between resolution in the radial and latitude directions. The choice of these parameters depends on the properties of the observed mode set, particularly the errors in the data; finding the best parameters for a given case often requires a substantial amount of trial and error, although in some cases a more systematic approach has been used.

For convenience, we group the methods into two broad categories: the "least-squares" methods, which aim to find a solution that fits the data; and the "localized averages" methods, which aim explicitly to construct averaging kernels that are localized in space and hence to obtain localized averages of the rotation rate in different parts of the solar interior. For reference, the methods are briefly summarized in Table 1.

\subsection{Least-Squares Methods}

These methods aim to find a solution that fits the data, by

\begin{tabular}{|c|c|}
\hline Method & Brief Description \\
\hline 2dRLS & Two-dimensional Regularized Least Squares \\
\hline $1.5 \mathrm{dRLS}$ & 1.5-dimensional Regularized Least Squares \\
\hline OMD . & 1.5-dimensional Regularized Least Squares with Optimal Mesh Distribution \\
\hline 2dSOLA & Two-dimensional Subtractive Optimally Localized Averages \\
\hline $1 \mathrm{~d} \times 1 \mathrm{dOLA}$ & Approximate Two-dimensional Multiplicative Optimally Localized Averages \\
\hline $1 \mathrm{~d} \times 1 \mathrm{dSOLA} \ldots . .$. & Approximate Two-dimensional Subtractive Optimally Localized Averages \\
\hline $1.5 \mathrm{dSOLA} \ldots \ldots \ldots$ & 1.5-dimensional Subtractive Optimally Localized Averages \\
\hline
\end{tabular}
means of a regularized least-squares (RLS) fit. RLS methods generally work by minimizing the sum of the $\chi^{2}$ residual of the fit and a function penalizing undesirable features in the solution. The regularization term often takes the form of an integral of the square of some derivative of the solution, since this allows efficient algorithms to be used.

The two-dimensional regularized least-squares method (2dRLS) makes a simultaneous fit to all the data using a

TABLE 1

Brief Summary of the Seven Inversion Procedures Used, Details of Which Are in $§ 3$ 
function that is piecewise bilinear on a rectangular mesh in the $(r, \theta)$ plane. Among all such functions, the 2dRLS solution is the one that minimizes the sum of the $\chi^{2}$ fit to the data plus two integral terms that penalize functions with large second derivatives with respect to $r$ or $\theta$. Full details of the method and its implementation are given by Schou et al. (1994). For all the 2dRLS inversions presented in this paper, we have used a mesh with 24 uniform intervals in $\theta$ and 100 nonuniform intervals in $r$; the radial mesh was chosen to be approximately uniform in acoustical radius, such that the intervals in $r$ are smaller near the surface.

The 1.5-dimensional regularized least squares (1.5dRLS) expands the rotation rate $\Omega(r, \theta)$ as in equation (12) and solves each of the resulting independent inversion problems in one dimension (the radial direction) by means of an RLS technique with iterative refinement (cf. Antia, Chitre, \& Thompson 1996). This technique minimizes the sum of squared differences for each coefficient together with the integral term that penalizes functions with large second derivatives with respect to $r$. A cubic B-spline basis function on a set of 54 almost uniformly spaced knots is used to represent the radial dependence. The method of iterative refinement effectively chooses appropriate smoothing for each component, and it is found that the optimum value of smoothing increases with the order of the splitting coefficient. The results from each one-dimensional inversion are then combined to obtain a representation of the full twodimensional rotation profile.

A second 1.5-dimensional, or semiparameterized, formulation (optimal mesh distribution) is based on the expansion in equation (12) of the angular velocity and the ClebschGordan expansion of the rotational splittings (Ritzwoller \& Lavely 1991). The optimal mesh distribution (OMD; EffDarwich \& Pérez-Hernández 1997) computes an optimal distribution for the inversion mesh points in the radial direction and then uses an RLS technique, with a secondderivative smoothing constraint.

\subsection{Localized Averages Methods}

These methods explicitly form linear combinations of the data and corresponding kernels such that the resulting averaging kernels (cf. eq. [9]) are, to the extent possible, localized near the target positions, through appropriate choice of the coefficients $c_{i}\left(r_{0}, \theta_{0}\right)$ (see Backus \& Gilbert 1968).

In the two-dimensional subtractive optimally localized averages method (2dSOLA), the goal is to approximate $\mathscr{K}$ to a prescribed target $\mathscr{T}\left(r_{0}, \theta_{0}, r, \theta\right)$ by minimizing

$$
\begin{aligned}
\int_{0}^{R} \int_{0}^{\pi / 2}\left[\mathscr{T}\left(r_{0}, \theta_{0}, r, \theta\right)-\mathscr{K}\left(r_{0}, \theta_{0}, r, \theta\right)\right]^{2} r d r d \theta \\
+\lambda \sum_{i=1}^{M}\left[\sigma_{i} c_{i}\left(r_{0}, \theta_{0}\right)\right]^{2},
\end{aligned}
$$

subject to $\mathscr{K}$ being unimodular (cf. Pijpers \& Thompson 1992, 1994). Here the first term ensures that the averaging kernel is close to the target form, while the second controls the error in the inferred solution, the trade-off between the two being controlled by the parameter $\lambda$. We have employed Gaussian targets, symmetrized around the equator. The radial width was chosen proportional to the local sound speed (e.g., Thompson 1993) while the width in latitude had a constant linear extent.

A simple implementation of the above method would require the factorization of one or more $M \times M$ matrices and hence would be prohibitively expensive computationally for two-dimensional inversion because $M$ is so large. We have applied an iterative Lanczos-type algorithm described by Larsen \& Hansen (1997), which furthermore takes advantage of the special structure of the kernels shown in equation (11) (see Larsen 1997). This has made it possible to use the full 2dSOLA method even for the large SOI-MDI data sets.

Other ways of making localized averages more tractable for our two-dimensional problem include the $\mathbf{R}^{1} \otimes \mathbf{R}^{1}$ methods, originally proposed by Sekii (1993a) and subsequently modified by Sekii (1993b) and Pijpers \& Thompson (1996). The methods use the separation of the kernels $K_{n l m}$ as in equation (11) to generate averaging kernels efficiently.

The $1 \mathrm{~d} \times 1 \mathrm{dOLA}$ method (Sekii 1993b, 1995) is similar to the 2dSOLA in that the solution is based on explicit determination of appropriate inversion coefficients $c_{i}\left(r_{0}, \theta_{0}\right)$. However, these are not sought in the full space. Instead, motivated by the near factorization of the splitting kernels, it is assumed that

$$
c_{i}=\tilde{c}_{n l} \beta_{l m},
$$

in the case of data in the form of individual splittings, with a similar expression for $a$-coefficients; here the (latitudinal) inversion coefficients $\left\{\beta_{l m}\right\}$ are determined in such a way that the first term of the angular part of the averaging kernel is localized (the SOLA method has been used for this purpose). Then the (radial) inversion coefficients $\left\{\tilde{c}_{n l}\right\}$ are determined by optimizing a localization criterion in two dimensions including the second term in equation (11) (see Sekii 1995 for details). However, the second term is still ignored in the determination of $\beta_{l m}$. This is different from Sekii (1993a), who entirely ignored the second term in the process of determining the inversion coefficients.

In contrast, the $1 \mathrm{~d} \times 1 \mathrm{dSOLA}$ method of Pijpers \& Thompson (1996) utilizes the full expression (eq. [11]) for the kernels throughout, including the process of the latitudinal inversion. In this $\mathbf{R}^{1} \otimes \mathbf{R}^{1}$ method, first linear combinations of the $G_{1}$ and linear combinations of the $G_{2}$ are localized in latitude simultaneously, for every $l$. After this, linear combinations of the radial functions $F_{R}=F_{1}+\zeta F_{2}$ are localized in radius, where $\zeta$ is a factor that depends on $n$ and $l$ but not on $r$ or $\theta$. The linear coefficients thus obtained, when combined with the original kernels, then produce two-dimensional averaging kernels that are well localized both in radius and in latitude. As in all SOLA-type methods, the free parameters are resolution widths in radius and in latitude for the Gaussian target functions usually used, and error-weighting factors (which we set to zero for the latitudinal parts of the inversion).

Finally, another inexpensive approach to using localized averages is in a semiparameterized inversion. Our 1.5dSOLA method takes the data in the same form as in the $1.5 \mathrm{dRLS}$ method above but uses an SOLA inversion in the radial direction. The trade-off parameter is rescaled at each target location to keep constant the ratio between the spatial resolution (computed from the optimal mesh distribution of the OMD) and the width of the averaging kernels for each target location (Eff-Darwich 1998).

\section{RESOLUTION AND RELIABILITY OF ROTATIONAL INVERSIONS}

Before presenting results of inverting the SOI-MDI rota- 
tional splitting data, we first assess the resolution and reliability of inversion solutions obtained from this data set. It follows from equation (8) that the solution $\bar{\Omega}\left(r_{0}, \theta_{0}\right)$ can be regarded as an average of the true $\Omega(r, \theta)$, weighted by the averaging kernel, plus an error term $\sum c_{i} \epsilon_{i}$ coming from the noise $\epsilon_{i}$ in the data. The resolution is therefore completely described by the averaging kernel. The averaging kernel depends on various factors: the data set (through the data kernels) although not the data values, the inversion method, the chosen values of the trade-off parameters, and the data uncertainties (through their influence on the choice of tradeoff parameters). Averaging kernels for three of the inversion methods are shown in Figure $1 a$ at five target locations and in Figure $1 b$ in close-up at four further locations. Over a substantial portion of the $\left(r_{0}, \theta_{0}\right)$ plane the averaging kernels have a substantial peak near the target position $\left(r_{0}\right.$, $\theta_{0}$ ); moreover, most of the contribution to the integral of the averaging kernel comes from that peak, although there may be other structure in the averaging kernel further away from the target location. In such a case, when the kernel can therefore be said to be localized about the target location, we can consider the radial and latitudinal widths of this principal peak in the averaging kernel to be measures of the radial and latitudinal resolution of the inversion. These are of course functions of $\left(r_{0}, \theta_{0}\right)$. Obviously these positions and widths do not capture all the details of the averaging kernels. In particular, some kernels have significant negative sidelobes. The effect of such features depends strongly on the true underlying rotation rate. RLS methods, for instance, tend to produce averaging kernels that will preserve a linear function of radius (because of the regularization term). If the true rotation rate is indeed of this form then negative sidelobes will have no serious influence. If, on the other hand, the rotation rate has sharp features where the negative sidelobes are located, significant problems can occur. Methods producing kernels with smaller features far from the target position (e.g., OLA methods) are not susceptible to this problem but tend to have wider main peaks.

Although the averaging kernels contain all the information about the resolution, it is often easier to obtain an overall impression of the resolution of the inversion by applying the same technique to artificial data generated for a known rotation profile. We have therefore performed a hare-and-hounds exercise in which one author invented two artificial rotation profiles (which were not revealed to the other authors). He then computed artificial splitting data, according to equation (7), corresponding to the available SOI-MDI data, and added to these random Gaussian noise with the same standard deviations as those estimated for the noise in the real data. These were then inverted independently by seven groups of other authors, using independent inversion methods as described in the previous section. The inverters drew their own independent conclusions about the unknown rotation profiles. Subsequently they then intercompared their results and inferences and agreed on a set of combined conclusions. Finally, the true profiles were made known and the inversion results and conclusions were tested against the "truth."

A hare-and-hounds exercise such as this does more than simply illustrate the resolution of the methods. Because it was conducted in blind fashion, with the inverters not knowing what the true profiles looked like, it also tested the inverters' judgement in choosing the appropriate trade-off values and in interpreting the results of their inversions: in particular, how much faith to place in bumps, dips, and wiggles that might be genuine features of the rotation profiles or might arise simply from noise in the data. Under the assumption that the noise in each datum is independent, normally distributed with zero mean and known variance, the error $\sum c_{i} \epsilon_{i}$ in the solution $\Omega\left(r_{0}, \theta_{0}\right)$ is also normally distributed with zero mean and with variance given by equation (10). Part of the job of the inverter is to make the appropriate trade-off between improving the resolution and reducing the variance of the error. The errors at neighboring points will in general be correlated (cf. Schou 1991b; Howe \& Thompson 1996) so that data noise gives rise in the solution to bumps, dips, and wiggles corresponding to the scale over which neighboring points have positively correlated errors. This scale is generally similar to the resolution scale defined by the widths of the averaging kernels, which makes identifying the reality of such features problematic. Any feature whose amplitude is only a few error standard deviations must therefore be treated with great caution. An aspect of the real data that is not tested by the current hare-and-hounds exercise is the possibility that the values of the data errors $\epsilon_{i}$ contain a systematic component (i.e., that they do not have zero mean), that they are correlated, or that their variances have been incorrectly estimated.

While the systematic errors are hard to estimate, some estimates have been made of the magnitude of error correlations. Within a multiplet the covariance matrix of the parameters is estimated internally as part of the fitting (Schou, Christensen-Dalsgaard, \& Thompson 1995). The correlations between adjacent odd $a$-coefficients (e.g., $a_{1}$ and $a_{3}$ ) for medium-degree modes are of the order 0.1 for most modes, but for some modes up to 0.25 . The error correlations for modes with different $n$ or $l$ are not estimated in the fitting procedure. However, such correlations are expected to be very low for most modes, owing to their spacings being large compared with the line widths and compared with the size of the frequency intervals used for the fitting. For closely spaced modes (e.g., adjacent modes at the high-l end of each ridge) one might expect some correlations. We thus conclude that correlations are unlikely to be a problem for the vast majority of modes and that the assumption of independent errors is therefore likely to be a good approximation.

The results of the hare-and-hounds exercise are presented in Appendix A. Here we consider only one of the principal conclusions, regarding the region of reliability of inversions of the present SOI-MDI data. In the region where the solution can be believed one certainly expects that the main peak of the averaging kernel should be close to the desired point, namely the target location. In Figure 2 we illustrate how far the actual local maximum of the averaging kernels is from the intended target location, as a function of target position inside the Sun, for the 2dSOLA method.

There is a fairly substantial region including the equatorial and mid latitudes in the convection zone, where the kernels are peaked essentially where they should be. However, the kernels become poorly localized in the deep interior and near the poles: only modes of relatively low degree penetrate the deep interior, and likewise only modes of azimuthal order close to zero feel the near-polar rotation, so that kernels $K_{n l s}^{(a)}$ for the $a$-coefficients (cf. eq. [6]) fall off asymptotically as $\sin ^{3} \theta$ near the poles (Pijpers 1997). Since the first maximum in the mode kernels moves closer to the 


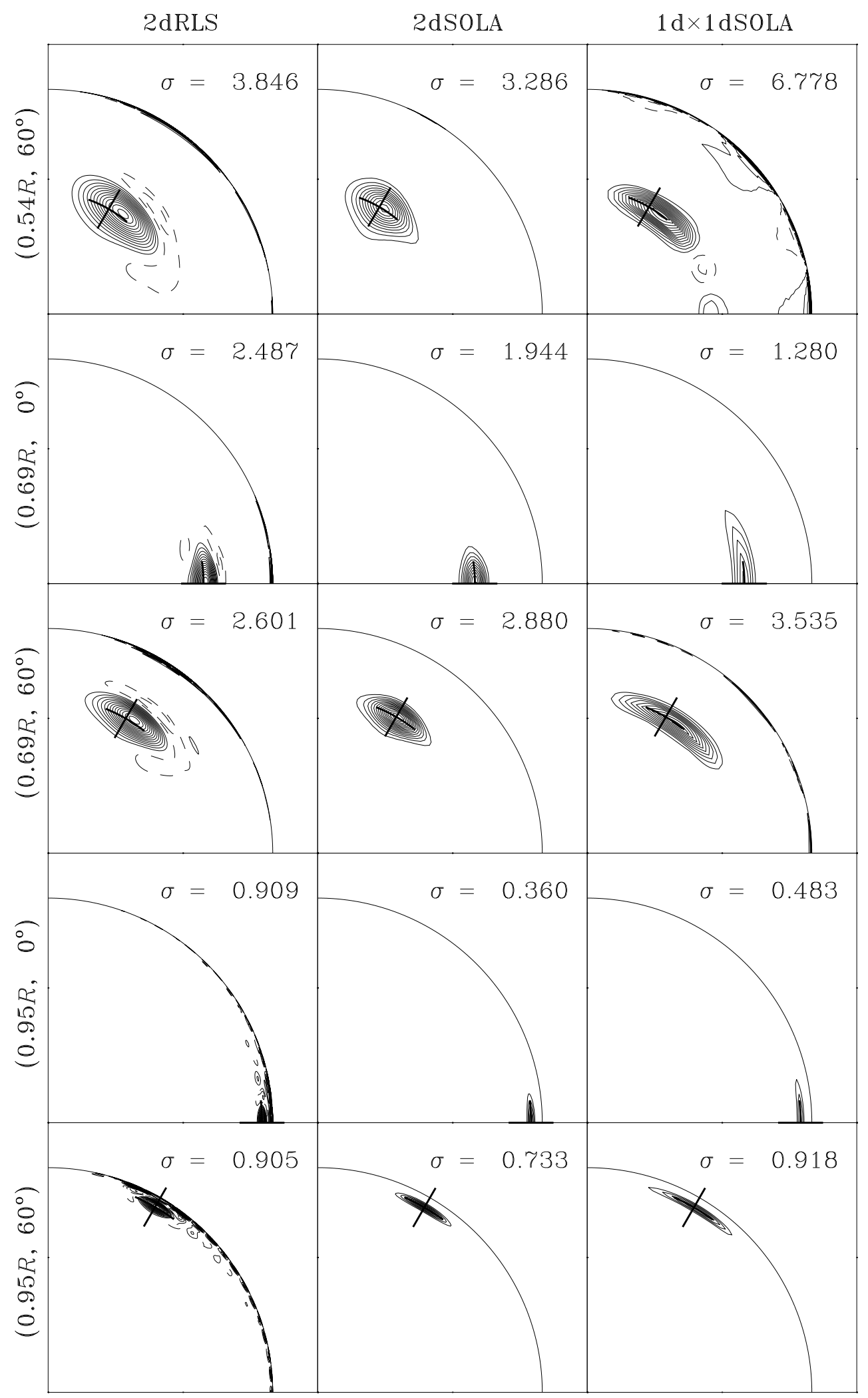

FIG. $1 a$

FIG. 1.-(a) Selection of averaging kernels, at five target locations within solar $(r, \theta)$ quadrants (equator: bottom right; pole: top left). The columns correspond to three different inversion methods: from the left they are 2dRLS, 2dSOLA, and $1 \mathrm{~d} \times 1 \mathrm{dSOLA}$. The rows correspond to the different target locations (crosses): from the top they are at radius and latitude $\left(0.54 R, 60^{\circ}\right),\left(0.69 R, 0^{\circ}\right),\left(0.69 R, 60^{\circ}\right),\left(0.95 R, 0^{\circ}\right)$, and $\left(0.95 R, 60^{\circ}\right)$. At a given target location the same contour spacing is used for all methods. The standard error $\sigma$ (in $\mathrm{nHz}$ ) at each target point has been indicated. (b) Enlargements of a selection of averaging kernels, at four near-surface target locations. The rows correspond to the different inversion methods: 2dRLS (top), 2dSOLA (middle), and $1 \mathrm{~d} \times 1 \mathrm{dSOLA}\left(\right.$ bottom). Target locations are (left to right) at radius and latitude $\left(0.95 R, 74^{\circ}\right),\left(0.99 R, 0^{\circ}\right),\left(0.99 R, 44^{\circ}\right)$, and $\left(0.99 R, 74^{\circ}\right)$. At a given target location the same contour spacing is used for both methods. 


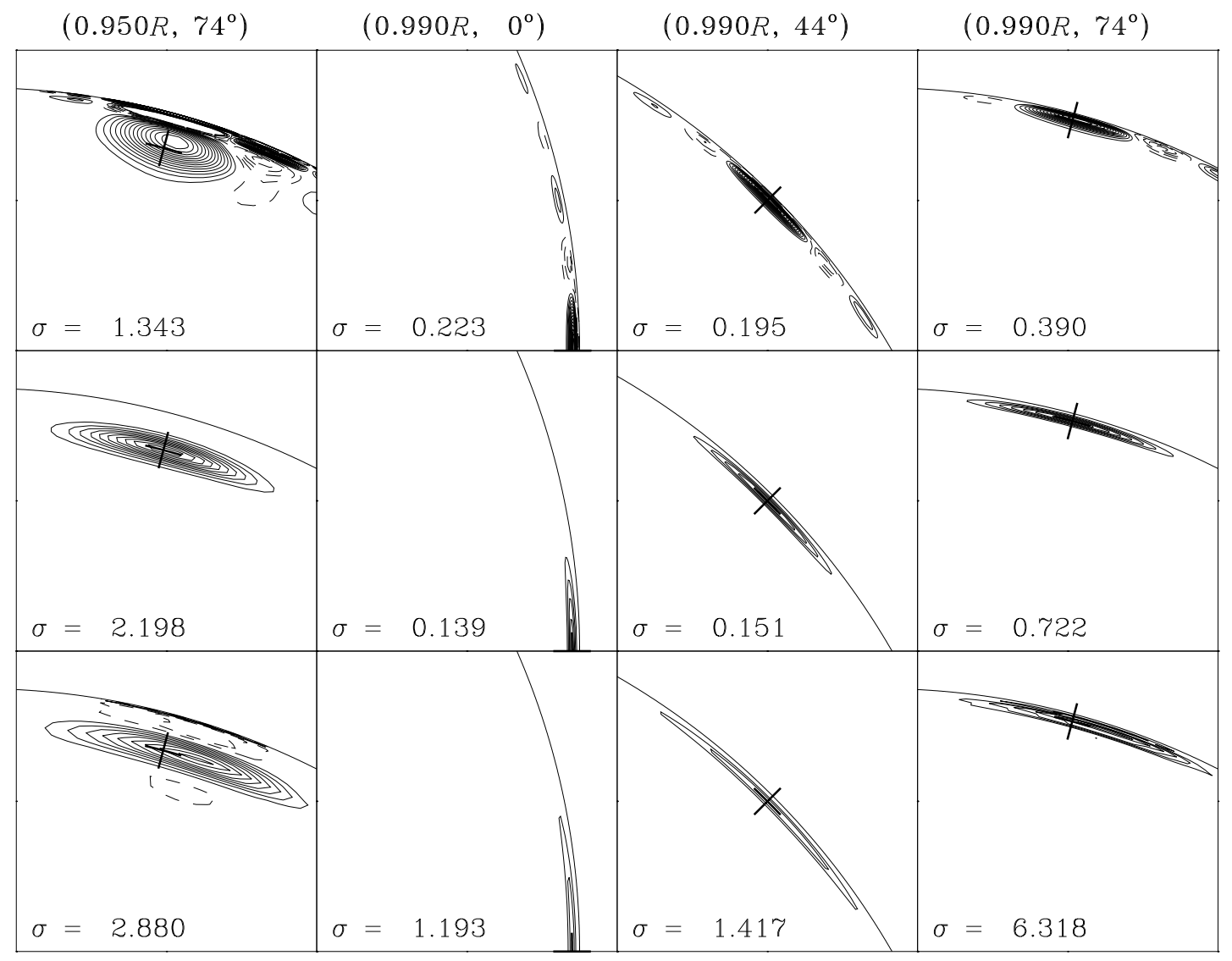

FIG. $1 b$

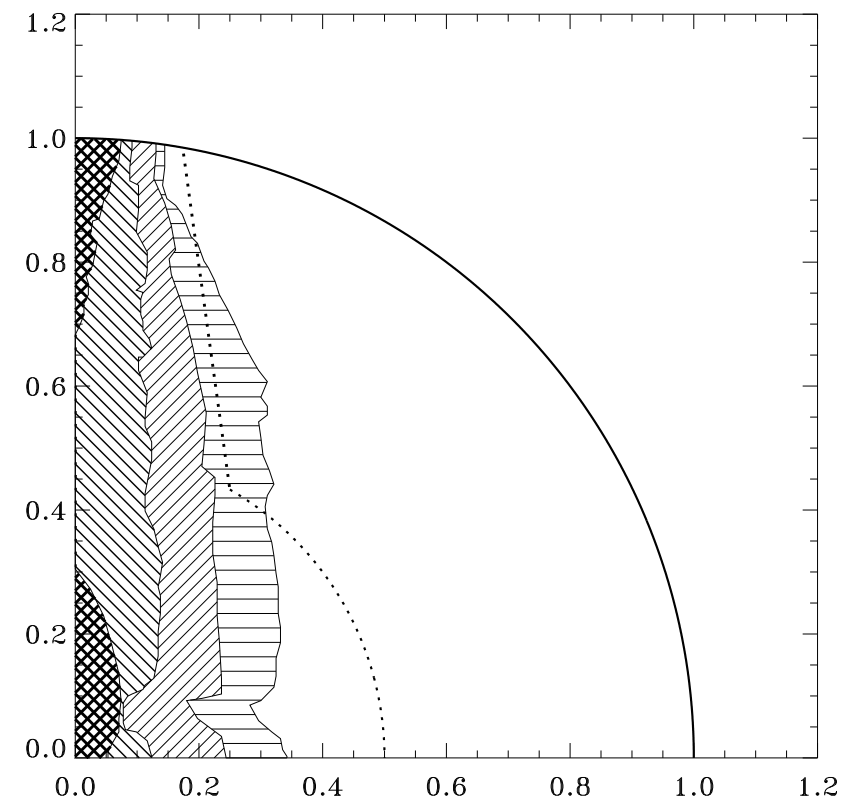

FIG. 2.-Shift of the location of the averaging kernels, relative to the target position, for 2dSOLA inversion. The properties of the averaging kernels are obtained by fitting symmetrized Gaussians, and the shift is measured in units of the extent of the kernels. The regions indicate a shift of less than 10\% (blank), 10\%-25\% (horizontally hatched), 25\%-50\% (southwest-northeast hatched), 50\%-100\% (northwest-southeast hatched), and more than $100 \%$ (cross-hatched). The dotted curve indicates the limit of reliability of the solution, discussed in the text and indicated in Figs. 3 and 4 by a shaded region. poles as $s^{-1}$, higher $a$-coefficients are better at probing high latitudes. However, the envelope of the kernels vary as $(\sin \theta)^{3 / 2}$, so even with a large number of $a$-coefficients it is still difficult to localize averaging kernels close to the poles.

One possible criterion for well-localized kernels is that the peak of the local maximum should be within one half the radial/latitudinal interquartile width (i.e., the distance between the first and third quartiles) of the target location. This criterion may be used to delimit the region where results seem to be reliable, i.e., essentially an image of the underlying rotation rate but blurred out by the finite resolution and only modestly affected by the data noise. For the present data set and based on the 2dRLS results (which give us a more conservative region than the 2dSOLA) this region, illustrated in Figure 2, is approximately as follows: target radii $r_{0} \geq 0.5 R$, equatorward of a line that goes from about $60^{\circ}$ latitude at $0.5 R$ to about $80^{\circ}$ latitude at the surface.

The precise details of that figure are not invariant if, for example, we were to renormalize the averaging kernels by choosing integration variables other than those in equation (8). Nonetheless, the good region we thus identify is consistent with that region where the hare-and-hounds inferences were reliable (cf. Appendix A), so that this seems to be a not unreasonable way of identifying the region of reliability. By design, the OLA methods produce better localized kernels than RLS methods. However, even OLA methods cannot localize kernels at target locations much beyond this region, as can be seen in Figure 2. 
We also point out that comparing the results from so many methods also gives some feel for the inversions' reliability, both for the hare and hounds and for inversions of SOI-MDI data. The region where the inversion methods are in good agreement is essentially the one described above.

\section{RESULTS OF INVERTING SOI-MDI DATA}

We have applied the inversion procedures described in $\S 3$ to the SOI-MDI frequency-splitting data. The same methods were applied to the artificial data in the hare-andhounds exercise, described in Appendix A. The trade-off parameter values were the same for inversions of both solar and artificial data, except for the 1.5dRLS where the smoothing is chosen automatically and so cannot be made exactly the same in the two cases.

\subsection{Large-Scale Differential Rotation}

The overall inferred rotation rates from four of the methods (2dRLS, 2dSOLA, 1.5dRLS, and $1 \mathrm{~d} \times 1 \mathrm{dSOLA})$ are illustrated in Figure 3. Regions where the solutions are deemed unreliable have been shaded, according to the criterion discussed above. The associated formal 1 standard deviation errors are shown in Figure 4. Figure 5 presents an alternative color representation of the inferred angular velocities.

The rotation is indeed roughly independent of radius through the bulk of the convection zone rather than constant on cylinders (cf. § 1). At low latitudes, however, there is a tendency toward rotation on cylinders and, except in the outer layers, the rotation decreases gradually with increasing depth. At high latitudes, the angular velocity appears to
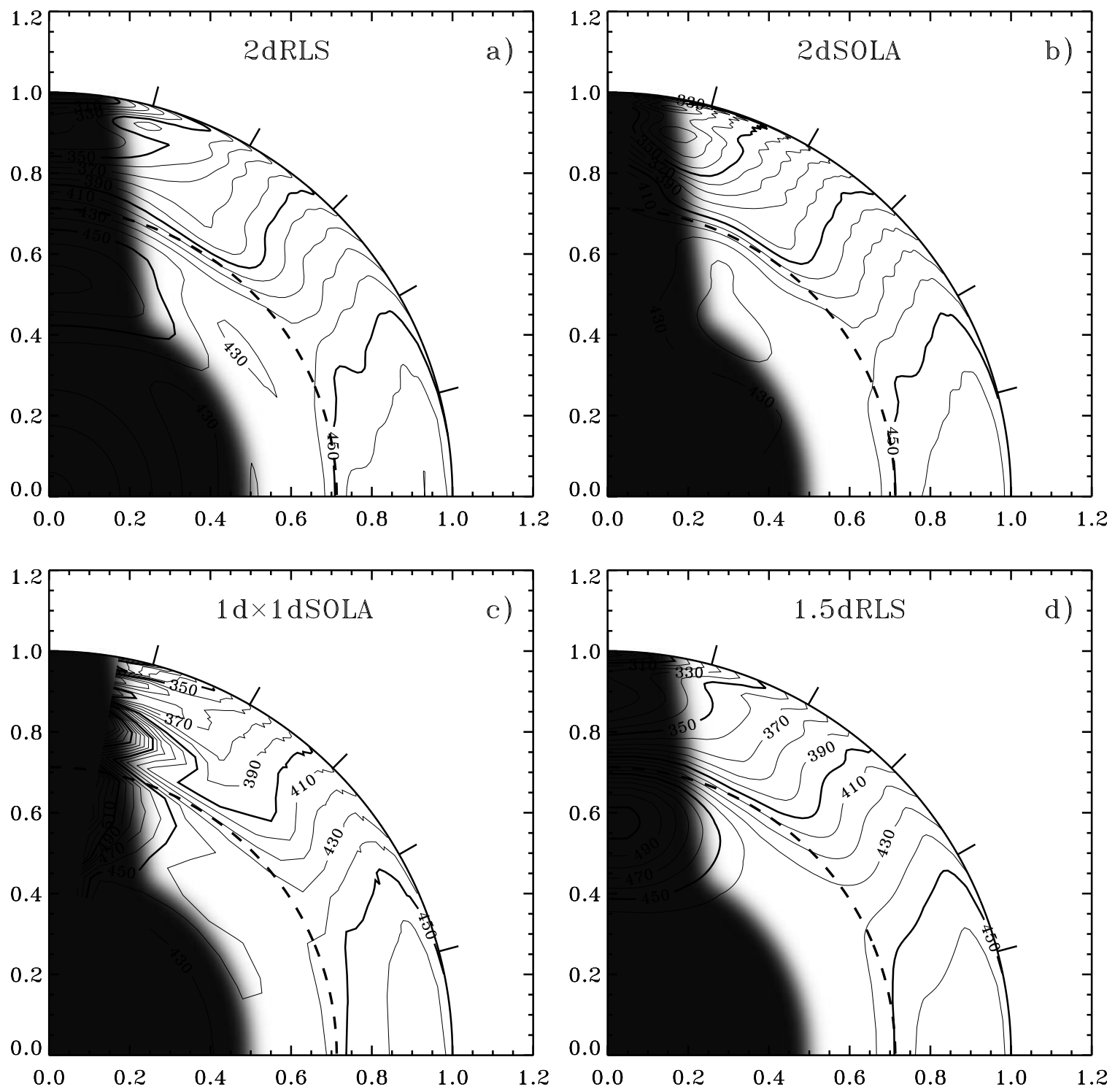

FIG. 3.-Inversions for rotation rate $\Omega / 2 \pi$ with radius and latitude for four inversion methods: (a) $2 \mathrm{dRLS}$; (b) $2 \mathrm{dSOLA}$; (c) $1 \mathrm{~d} \times 1 \mathrm{dSOLA}$; (d) $1.5 \mathrm{dRLS}$. Some contours are labeled in $\mathrm{nHz}$, and, for clarity, selected contours are shown as bold. The dashed circle indicates the base of convection zone, and the tick marks at the edge of the outer circle are at latitudes $15^{\circ}, 30^{\circ}, 45^{\circ}, 60^{\circ}, 75^{\circ}$. In such a quadrant display, the equator is the horizontal axis and the pole the vertical one, with the proportional radius labeled. The shaded area indicates the region in the Sun where no reliable inference can be made with the current data. 

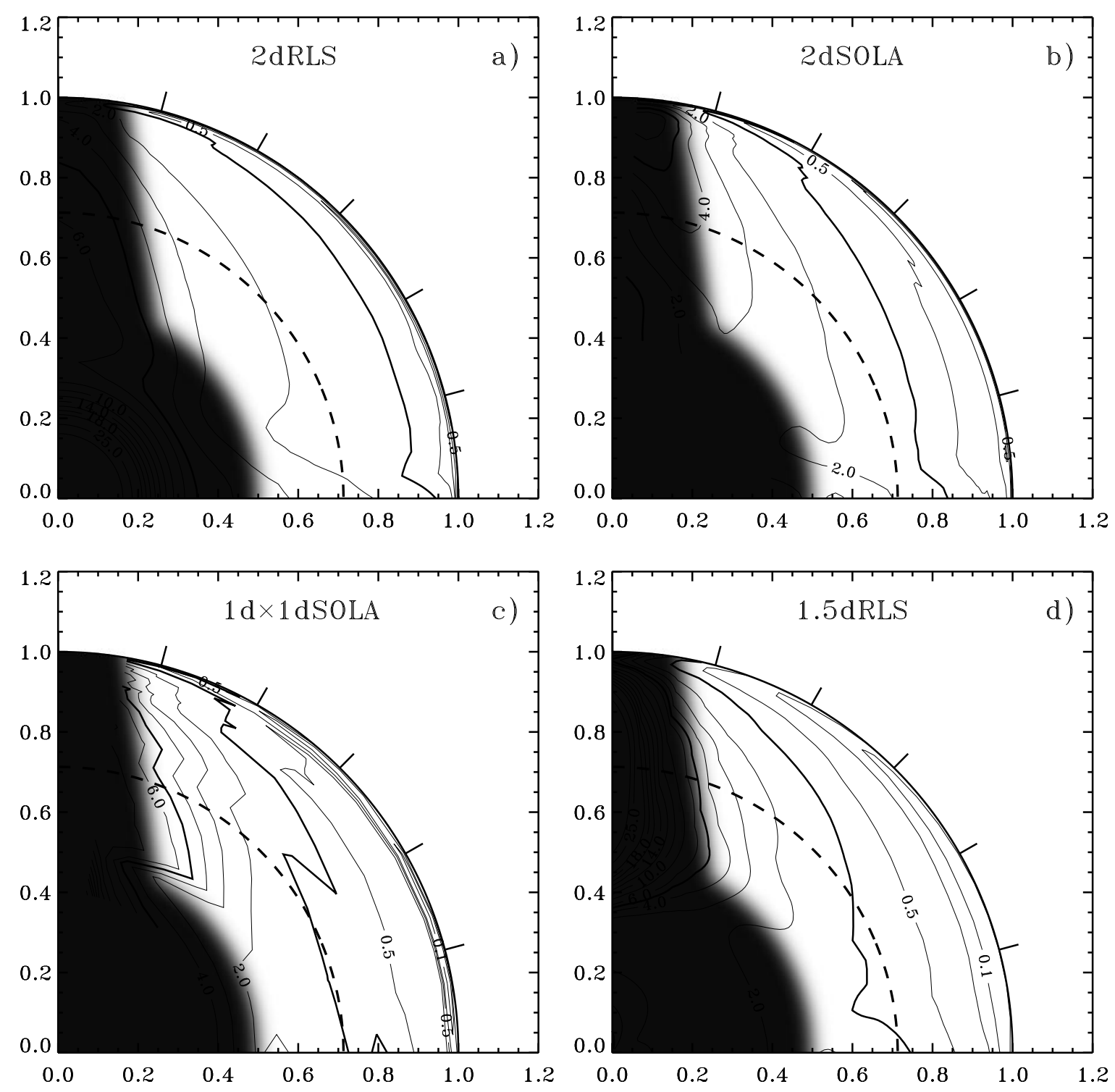

FIG. 4.-Contour plots of errors for same four cases as shown in Fig. 3. The contour levels used were (in nHz): 0.1, 0.2, 0.5, 1, 2, 3, 4, 5, 6, 8, 10, 12, 14, 16, $18,20,25$, and 30 . Selected contours are labeled by the error in $\mathrm{nHz}$. The style is otherwise the same as in Fig. 3.

increase with depth (see Fig. 7). These conclusions are broadly consistent with the picture of solar internal rotation that has emerged from previous helioseismic investigations (Thompson et al. 1996 and references therein). However, the quality of the SOI-MDI data is such that more detailed inferences are feasible. It is possible also with such data to make more reliable, well-localized inferences at high latitudes, as discussed in $\S 5.5$. The variation of $\Omega$ with latitude is shown in Figure 6, very close to the surface and at $0.95 R$. Inversion results are shown for all seven inversion methods listed in Table 1.

A fit to the measured Doppler surface rotation rate (derived by J. Beck 1997, private communication, from the results of Ulrich et al. 1988) is shown for comparison. The near-surface behavior is clearly in accordance with the observed surface rotation rate, and this is largely maintained down to $r=0.8 R$. It is evident that the solutions from different inversion methods are essentially consistent at low latitudes, while differing substantially at latitudes higher than $60^{\circ}$.

The surface latitudinal variation of the rotation has traditionally been represented by a three-term expression:

$$
\Omega_{s}=A+B \cos ^{2} \theta+C \cos ^{4} \theta
$$

(e.g., Snodgrass 1983). We have made a least-squares fit of this expression to the inferred angular velocity at $r=0.995 R$, limiting the fit to latitudes below $60^{\circ}$ where the solution appears most reliable. The resulting coefficients are presented in Table 2. The differences between the different fits and the fit derived from more traditional methods are relatively small: our purpose in performing the fits is primarily to look at the residuals to the fits, which are shown in Figure 8 and will be discussed in $\S \S 5.2,5.3$, and 5.5. Qualitatively, these residuals would be much the same whether our fits or the surface rotation rate obtained by traditional means were used when computing the residuals.

Radial cuts through the solutions, at selected latitudes $0^{\circ}$, $30^{\circ}, 60^{\circ}$, and $75^{\circ}$, are shown in Figure 7. Once again one can 

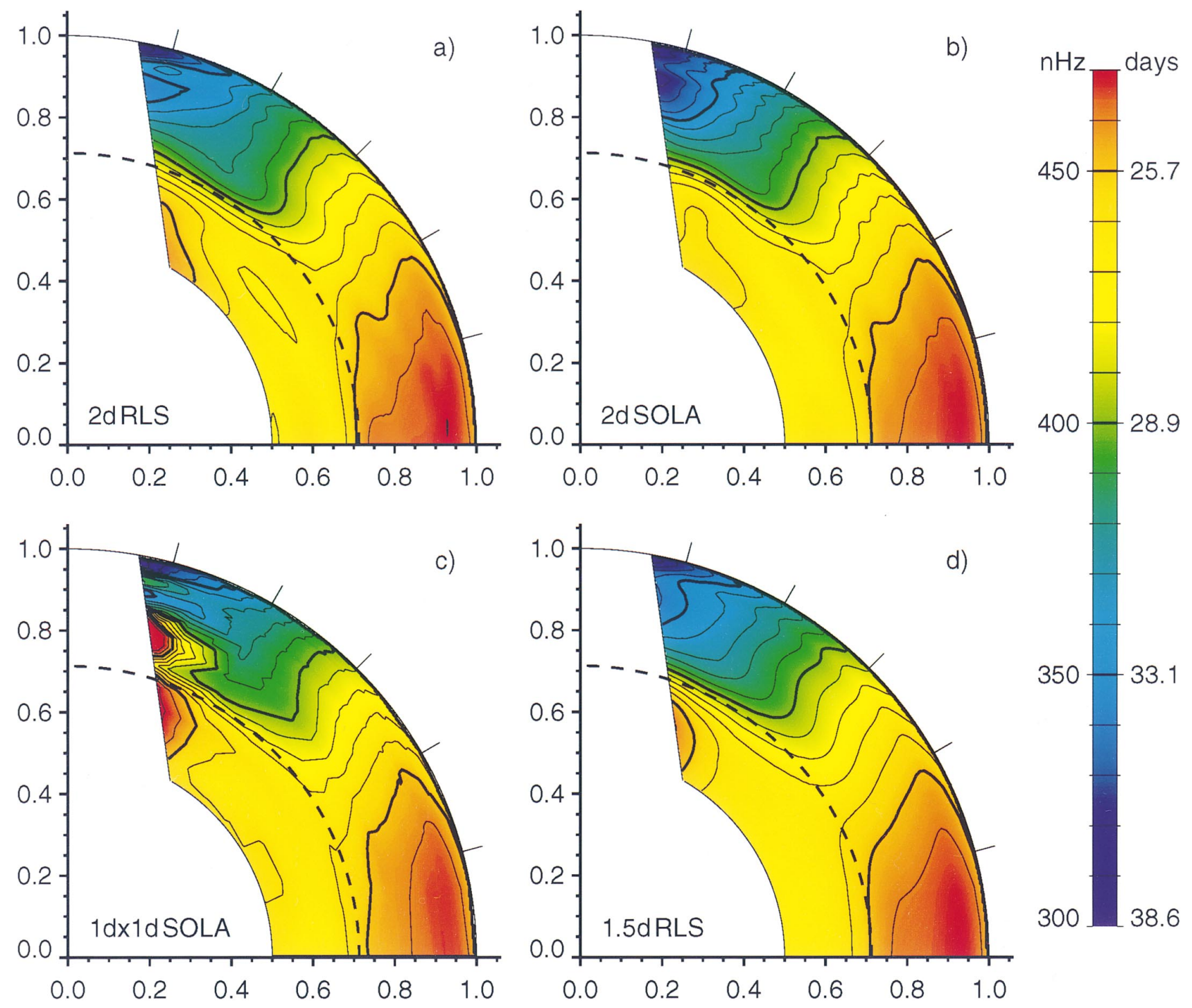

FIG. 5.-As a companion to Fig. 3, inversions for rotation rate $\Omega / 2 \pi$ with radius and latitude for four inversion methods: (a) $2 \mathrm{dRLS}$; (b) $2 \mathrm{dSOLA}$; (c) $1 \mathrm{~d} \times 1 \mathrm{dSOLA} ;(d) 1.5 \mathrm{dRLS}$.

see that for latitudes of up to about $60^{\circ}$, there is a very considerable degree of consistency between the results from different methods. The methods do not always agree to within the error bars; but there is no reason to expect that they necessarily should, because at any point the solution of

TABLE 2

The CoEFFicIENTS From A Fit of EquATION (17) To THE INFERRED Rotation Rate at RADIUS 0.995R, ACCORDING TO THE FOUR METHODS ILluUSTRATED IN Figure 3. Also Shown is the Rotation Rate FROM ULRICH ET AL. (1988).

\begin{tabular}{|c|c|c|c|}
\hline Method & $\begin{array}{c}A / 2 \pi \\
(\mathrm{nHz})\end{array}$ & $\begin{array}{c}B / 2 \pi \\
(\mathrm{nHz})\end{array}$ & $\begin{array}{c}C / 2 \pi \\
(\mathrm{nHz})\end{array}$ \\
\hline $2 \mathrm{dRLS}$ & 455.8 & -51.2 & -84.0 \\
\hline 2dSOLA & 455.4 & -52.4 & -81.1 \\
\hline $1 \mathrm{~d} \times 1 \mathrm{dSOLA}$ & 455.4 & -54.1 & -75.1 \\
\hline $1.5 \mathrm{dRLS} . . . \ldots$ & 455.1 & -47.3 & -85.9 \\
\hline Ulrich et al..... & 451.5 & -65.3 & -66.7 \\
\hline
\end{tabular}

each method is a differently weighted average of the underlying angular velocity (cf. § 1). The error bars indicate only how the particular method's solution would vary with a different realization of the data errors: they do not (unfortunately) give a probabilistic range in which the true value of the angular velocity is likely to lie. The maximum value of $\Omega, \Omega_{\max }=467.3-470.1 \mathrm{nHz}$, is found on the equator, at $r \simeq 0.93 R-0.94 R$; the ranges represent the spread in these values between the results of different inversion methods. Near the base of the convection zone (which is located at about $0.71 R$ ) there is a comparatively sharp transition to the radiative interior, where $\Omega$ varies little, if at all, around a value of about $430 \mathrm{nHz}$. There is also a shear layer, quite pronounced at lower latitudes, near the surface. We turn to this aspect of the rotation first.

\subsection{Subsurface Rotation Shear}

The solutions in Figure 3 indicate the presence of a radial shear in the outer parts of the convection zone, predominantly at low latitudes. Evidence for such a shear has been 


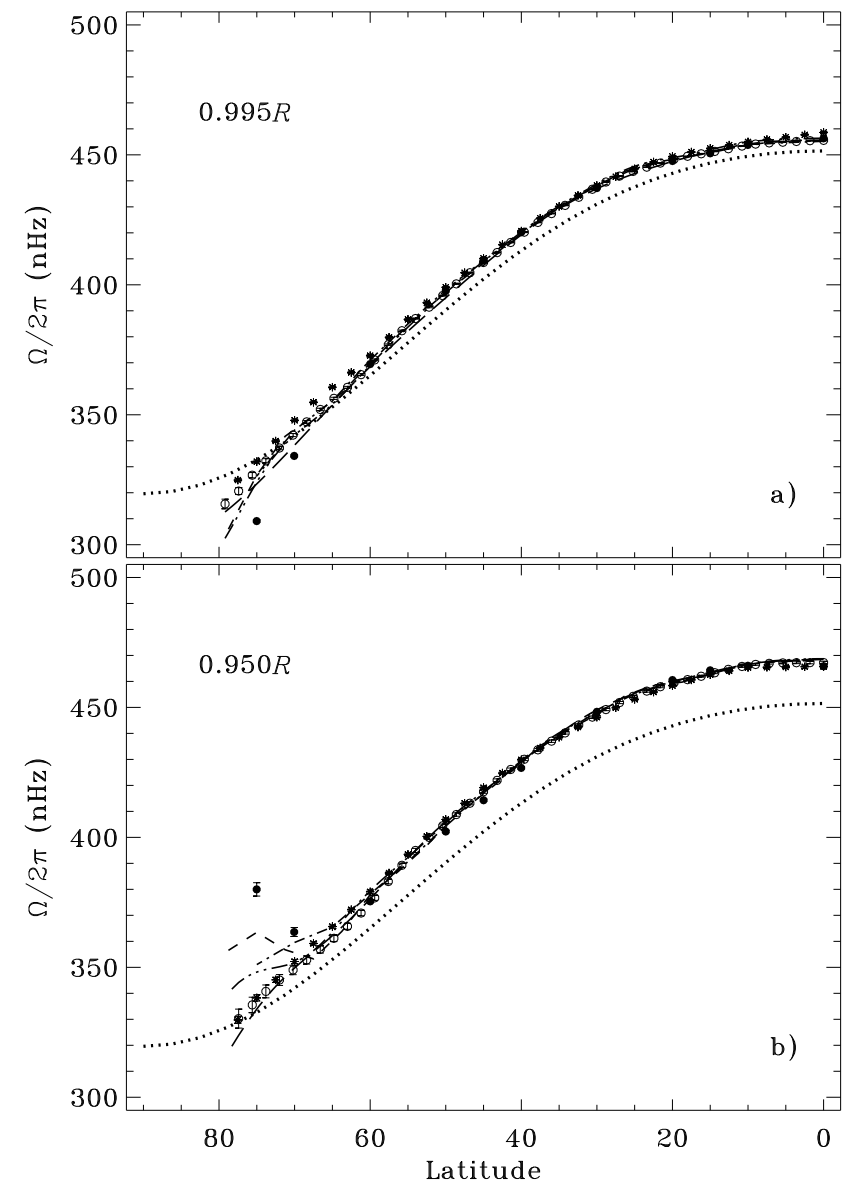

FIG. 6.-Latitudinal dependence of inferred rotation rate $\Omega / 2 \pi(a)$ close to surface and $(b)$ at $0.95 R$, compared with standard Doppler surface rates for various inversion methods. Line and symbol styles are 2dRLS (dashed line), 1.5dRLS (dot-dashed line), OMD (triple-dot-dashed line), 2dSOLA (open circles with $1 \sigma$ error bars), $1 \mathrm{~d} \times 1 \mathrm{dOLA}$ (stars with error bars), $1 \mathrm{~d} \times 1 \mathrm{dSOLA}$ (closed circles with error bars), and 1.5dSOLA (long-dashed line). The inferred solutions have been restricted to the region outside the shaded area in Fig. 3. The heavy dotted curve shows the fit to the surface rotation rate from Ulrich et al. (1988).

noticed previously (e.g., Rhodes et al. 1990). To illustrate the properties of this region in more detail, Figure 8 shows the residual, after subtracting the fitted $\Omega_{s}$ (eq. [17] and Table 2 ), as a function of $r$ at several latitudes. At the equator and at latitude $30^{\circ}, \Omega$ increases by about $10 \mathrm{nHz}$ below the surface, the maximum being near $r \simeq 0.95 R$. On the other hand, at $60^{\circ}$ there appears to be a decrease in $\Omega$ just below the surface, possibly followed by a slight increase. At $75^{\circ}$, where it is becoming difficult to localize averaging kernels, the results are more confused. The results from the different methods disagree by several standard deviations. A possible explanation for this is that the structure in some of the averaging kernels is picking up variations in the rotation rate far from the target location. Another, more disturbing, possibility is that the methods have different sensitivity to systematic errors. This is quite hard to test. The leastsquares methods (2dRLS and 1.5dRLS) agree with one another that there is a local minimum very near the surface and a strong increase in $\Omega$ beneath this. The $1 \mathrm{~d} \times 1 \mathrm{dSOLA}$ finds rather similar behavior, and the 2dSOLA also sees the increase at greater depth, although perhaps washed out by the averaging; but neither OLA method shows a very pronounced near-surface minimum.

To illustrate the near-surface behavior of the solution in more detail, Figure 9 presents an analysis of the 2dRLS results. The reason we choose to look in detail at this particular solution is that $2 \mathrm{dRLS}$ is the only one of our methods that attempts to fit the true rotation rate (rather than taking an average as do the OLA methods), in a manner that does not depend on an unregularized expansion in latitude. Figure $9 a$ shows the values of $\Omega$ (as inferred by $2 \mathrm{dRLS}$ ) at the maximum and, for latitudes above $50^{\circ}$, at the local minimum; they are given relative to the solution at $0.995 R$. The maximum is located near $0.95 R$ at all latitudes considered whereas the minimum is close to $0.99 R$. The high value of the maximum at about $70^{\circ}$ is associated with the submerged polar jet, discussed in $\S 5.5$.

The development of the local subsurface minimum is also reflected in Figure $9 b$, which shows an estimate of the radial gradient at $0.995 R$. It was evaluated from the difference in the solution at \pm 2 radial mesh points from this point, assuming independent errors. Although crude, this clearly illustrates the change in behavior between low and high latitude.

\subsection{Alternating Bands of Faster and Slower Rotation}

The residual rotation rate near the surface, after the three-term fit of equation (17) to the data has been subtracted, is illustrated in Figures 10 and 11 as a function of latitude. The dominant feature (Fig. 10) is evidently the sharp decrease, at latitudes above $70^{\circ}$, which is seen consistently by several independent methods. We return to this in $\S 5.5$. The top panel in addition shows results from an inversion of the f-mode data for the latitude dependence of rotation by Kosovichev \& Schou (1997). This inversion is sensitive to rotation at approximately the same radius of $r \simeq 0.995 R$ and does indeed show very similar features. When plotted on an expanded scale (Fig. 11), the residuals show small-scale spatial variations with a magnitude of 1 $\mathrm{nHz}$ corresponding to about $5 \mathrm{~m} \mathrm{~s}^{-1}$ and a scale of $10^{\circ}-15^{\circ}$. These variations were first reported from the MDI data by Kosovichev \& Schou, who referred to them as zonal flows. The investigation we report here is not, of course, independent of that work since we share the same f-mode data, but with the inclusion of p-mode splittings and inverting in both radial and latitudinal directions we can add to their findings some information about the depth dependence of these features. Although the alternating bands of faster and slower rotation are quite weak compared with the underlying rotation rate of about $450 \mathrm{nHz}$, they appear to be significant down to a depth of $0.01-0.02 R$ in several inversions. The $2 \mathrm{dRLS}$ results indicate that the flows may persist to a depth below the solar surface of perhaps as much as $0.05 R$, although the hare-and-hounds results lead us to view such an inference with some caution (see Appendix A).

\subsection{Structure of the Tachocline}

The rather abrupt transition near the base of the convection zone from latitudinally dependent, surface-like rotation to a nearly latitudinally independent rotation in the radiative interior is evident in Figure 3 and is even more clear in Figure 7. This transition (or at least that part of it belonging to the radiative interior; see $\S 1$ ) has been called the tachocline. The transition also shows up very clearly if one performs a one-dimensional inversion of the $a_{3}$ coefficient, as 


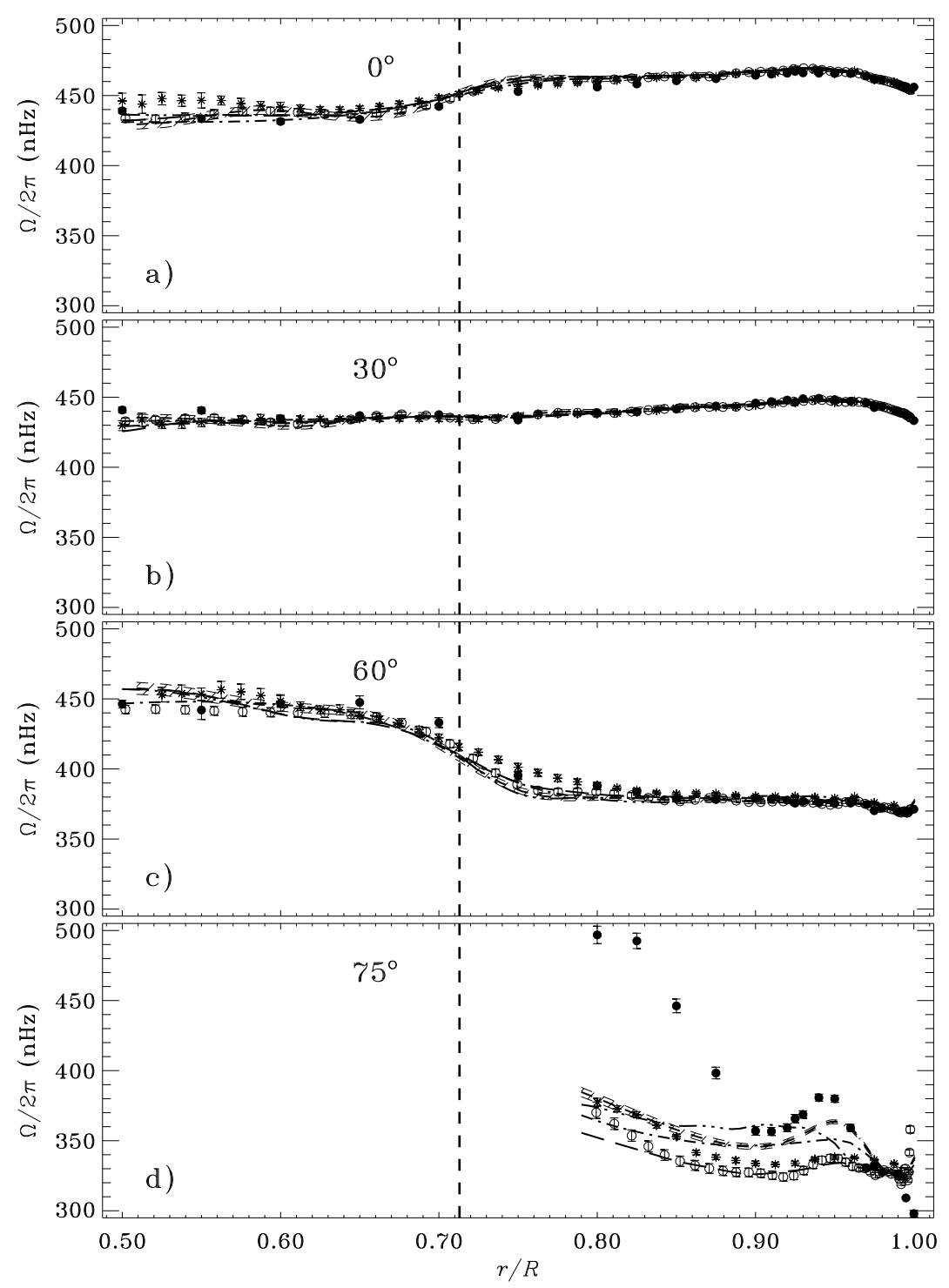

FIG. 7.- Radial dependence of the inferred rotation rate $\Omega / 2 \pi$ at constant latitudes, restricted to the region outside the shaded area in Fig. 3. Solutions are shown at the following latitudes: $(a) 0^{\circ}$ (equator); $(b) 30^{\circ} ;(c) 60^{\circ}$; and $(d) 75^{\circ}$. The line and symbol styles are the same as in Fig. 6 , with the addition for $2 \mathrm{dRLS}$ of a cross-hatched belt indicating the $1 \sigma$ error limits. The vertical dashed line indicates the base of the convection zone.

illustrated in Figure 12. Here 1dSOLA inversions of $a_{1}$ and $a_{3}$ have been carried out to determine the expansion functions $\Omega_{0}(r)$ and $\Omega_{1}(r)$, the latter being the leading $\theta$ dependent term in the expansion in equation (12), where the expansion functions $\psi_{2 s}^{(1)}$ given by equation (13) were assumed.

Kosovichev (1996) performed a similar analysis, and by fitting to the tachocline a functional form

$$
C_{1}+C_{2} \operatorname{erf}\left(\frac{r-r_{0}}{0.5 w}\right)
$$

where $C_{1}, C_{2}, r_{0}$, and $w$ are constants, $r_{0}$ and $w$ being, respectively, the position and width of the transition, he obtained a position of $r_{0}=0.692 R \pm 0.05 R$ and a width of $w=0.09 R \pm 0.04 R$. We have convolved a step of expression (18) with Gaussian approximations to the real averaging kernels and fitted them to the inferred rotation profiles at different latitudes between $r=0.5 R$ and $r=0.9 R$, thereby obtaining best-fit values of $r_{0}$ and $w$ as functions of latitude. Because we do not take into account the correlation between errors in the inferred profile at different nearby radii, we are cautious about quoting uncertainties. But from fitting both the 2dRLS and 2dSOLA inversions, it seems that at the equator and at quite high latitude $\left(60^{\circ}\right)$ the midpoint of the tachocline is $r_{0}=0.70 R-0.71 R$ and the thickness $w$ is indistinguishable from zero (i.e., no more than $w=0.05 R$ ). At latitude $15^{\circ}$ the fits suggest that the tachocline is slightly thicker, $w=0.05-0.1 R$, and possibly fractionally deeper by $0.005 R$. These results are consistent with the results of Charbonneau et al. (1998), Corbard et al. (1998), and Basu (1997), but are statistically less significant, owing presumably to the time series used in those investigations being substantially longer. At intermediate latitudes, like $30^{\circ}$ and $45^{\circ}$, the gradient across the tachocline is too small to determine a width with any reasonable accuracy.

At even higher latitudes (see Fig. 7) the transition in $\Omega$ to the value in the radiative interior occurs over a substantially 


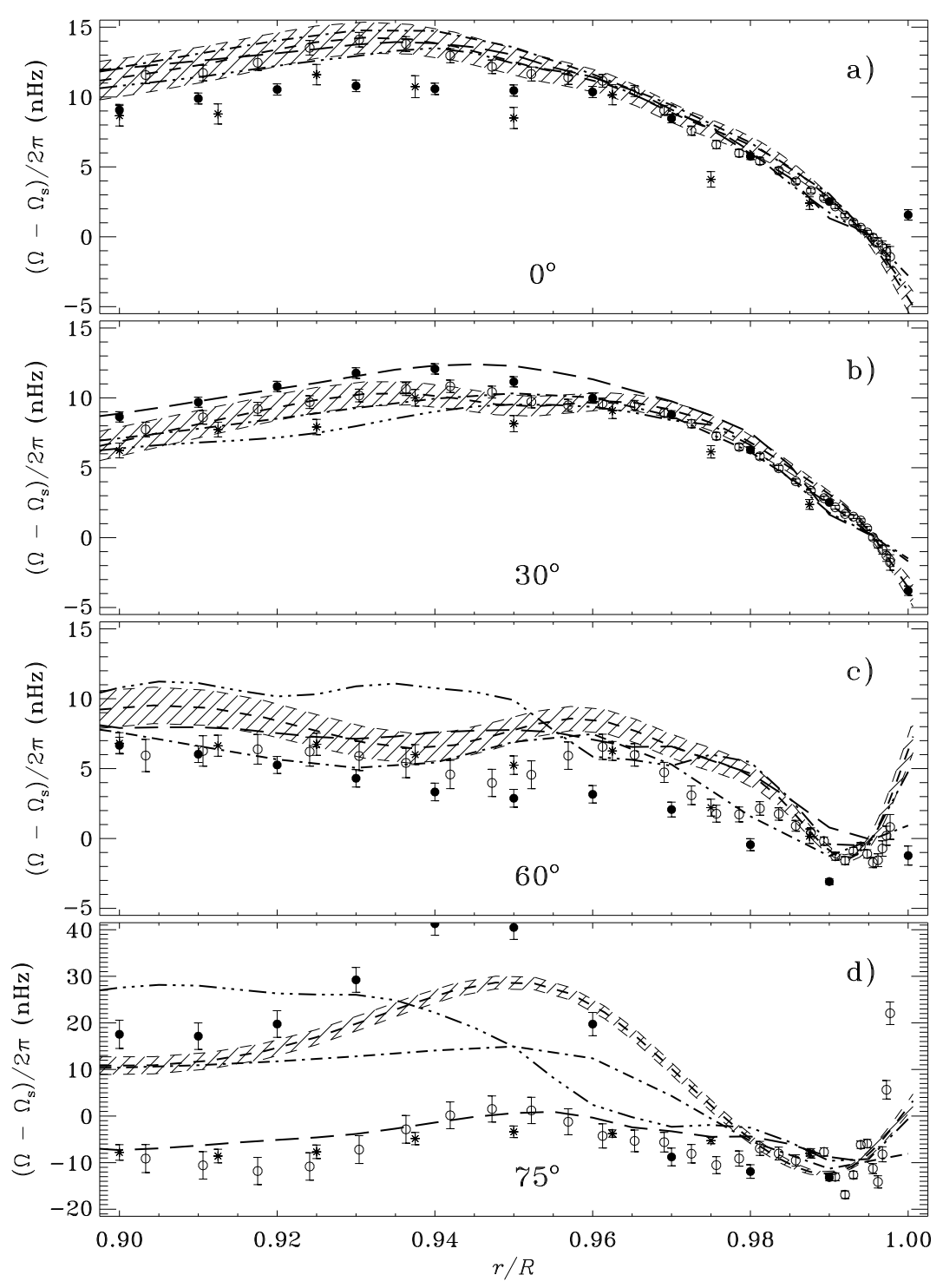

Fig. 8.- Residual $\left(\Omega-\Omega_{s}\right) / 2 \pi$ of the inferred rotation rate after subtraction of the near-surface fits (cf. Table 2 ), as a function of fractional radius in the outer $10 \%$, at fixed latitudes as labeled. The line and symbol styles are the same as in Fig. 7. Note that the vertical scale in the bottom panel corresponding to $75^{\circ}$ differs from the others.

broader region. Analyses of the averaging kernels and inversion of artificial data based on rotation that is discontinuous at the base of the convection zone have shown substantial degradation of resolution at latitude $75^{\circ}$, compared with lower latitudes. However, although further analysis is required, it is our impression that this effect may not be sufficient to account for the difference, between, say, the results at $60^{\circ}$ and those at $75^{\circ}$ latitude. We note that much of the transition at high latitudes appears to take place in the convection zone itself rather than at the base and in the subadiabatically stratified region. We therefore do not refer to this broad transition at high latitudes as part of the tachocline but rather consider it instead to be part of the pattern of differential rotation in the convective envelope.

\subsection{Polar Rotation and High-Latitude Submerged Jet}

As we have already commented, the deviation from the three-term fit of equation (17) to the inferred rotation rate shows interesting behavior at high latitudes. Indeed, the dominant feature of the residuals to the three-term fit, shown in Figure 10, is the sharp decrease at latitudes above $70^{\circ}$, which is seen consistently by several independent methods. Similar results are obtained from an inversion of f-mode data for the latitude-dependence of rotation (top panel), where the decrease at high latitude is again evident. While there is no reason to expect solar rotation to follow a simple relation such as equation (17), we nonetheless find this behavior striking.

Figures 3 and 7 show some other interesting near-surface behavior at high latitudes, particularly in the 2dRLS solution. At the outset we bear in mind that this is close to the limit of where the averaging kernels can be localized, and in regions of little data the 2dRLS method with secondderivative smoothing, like its one-dimensional counterpart (e.g., Christensen-Dalsgaard, Schou, \& Thompson 1990), tends to extrapolate the solution linearly from neighboring regions. This causes the solution in, e.g., Figure 3 to appear qualitatively different from, for example, the 2dSOLA solution. Even in view of these caveats, certain features appear to be shared by the results of several methods. These are 

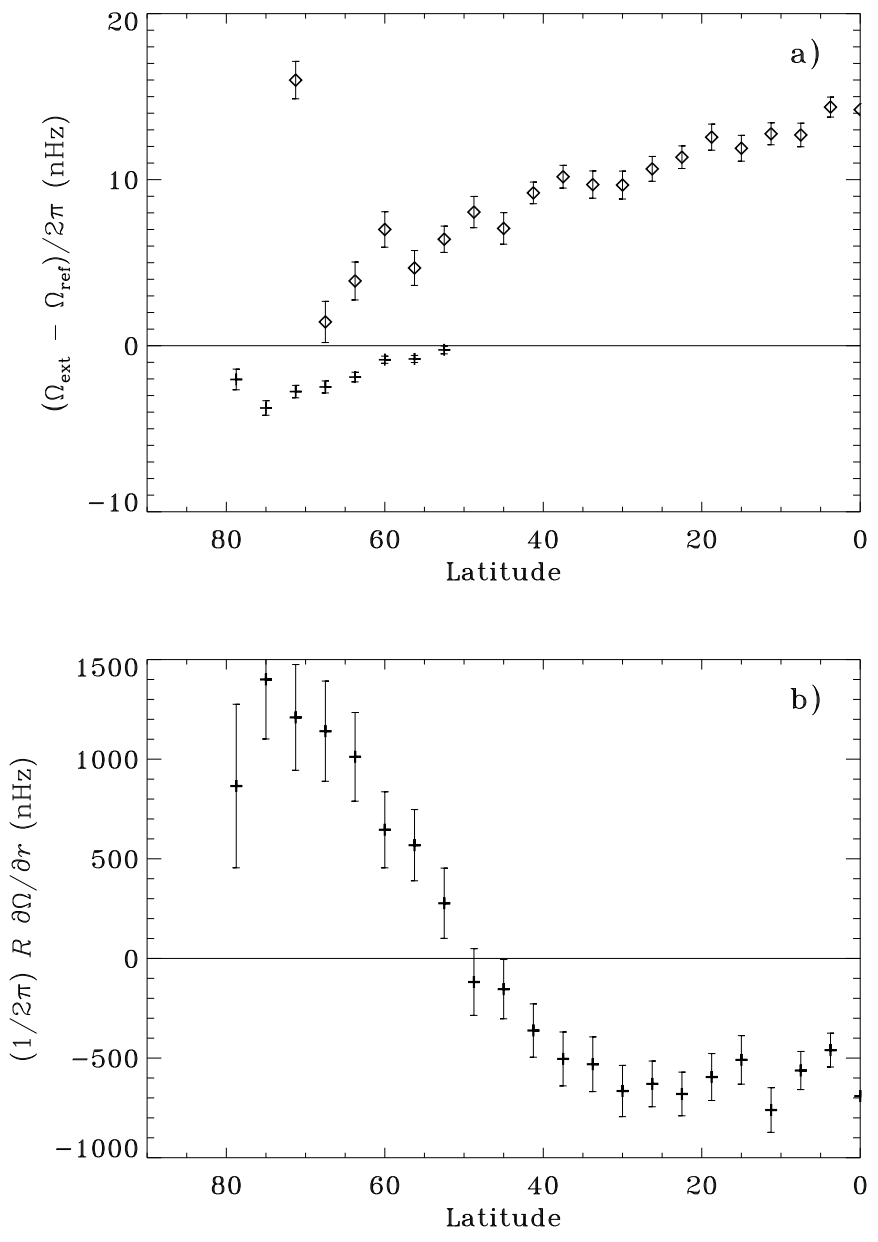

FIG. 9.- Near-surface extrema and radial derivative in the 2dRLS solution. (a) The value at the maximum around $0.95 R$ (diamonds) and the minimum around $0.99 R$ (crosses) in the solution, relative to the reference value at $0.995 R$. For latitudes below about $50^{\circ}$ the solution has no nearsurface minimum. (b) Estimate of the derivative of the inferred solution near $0.995 R$ and its $1 \sigma$ errors.

perhaps most clearly seen in the cuts at $75^{\circ}$ latitude in Figure 7. There is some evidence for a sharp decrease just below the surface. This is followed, in essentially all the results shown, by a maximum around $r=0.95 R$. According to the contour plot in Figure $3 a$, this may have the form of a localized, jetlike structure; the localization toward lower latitude is supported by the behavior of the maximum shown in Figure $9 a$. We note, however, that such a feature is not seen so clearly in the results of inversions by other methods. At somewhat greater depth there appears to be a broader minimum, also clearly visible in Figure 7.

To try to establish better whether the apparent jet is a real solar feature, we have looked at combinations of $a$ coefficients corresponding to different latitudes, in the manner of Wilson \& Burtonclay (1995) and Wilson, Burtonclay, \& Li (1996) (cf. Fig. 2 of Thompson et al. 1996). This is tantamount to making a kind of inversion in the latitudinal (but not the radial) direction. By plotting the combinations of coefficients against the mode turning point, one can see by eye the depth-averaged rotation felt by the modes. Viewing the data in this way shows that they do clearly contain some signal of an increased rotation rate at $r \simeq 0.95 R$. Not readily apparent from such an analysis is how well localized in latitude the enhanced rotation rate is (i.e., whether it is a jet or a ridge), since the data combinations are far from perfectly localized in latitude. (If it were otherwise, we would always do this, in preference to our more expensive full two-dimensional inversions!) Nor does it answer the question of whether it is a real solar feature or a data artifact. Nevertheless, it is reassuring and significant that it can be thus seen in the raw data.

We reiterate that any local inferences become unreliable at sufficiently high latitudes. This is borne out by the differences between the results obtained by different methods, as is illustrated in the $75^{\circ}$ panel of Figure 7, which are substantially larger than the estimated errors in the results and must be due in part to the differences in the averaging kernels of the methods when the data are pushed to their limits. In particular the 2dRLS and 2dSOLA results, while similar in shape, are shifted by about $20 \mathrm{nHz}$ relative to each other. In contrast, in the corresponding results obtained with artificial data (hare-and-hounds case test2; see Fig. 19 in the Appendix), where the inferred rotation rate was similar to the solar rate, no such differences were found, even though the random errors in the artificial data were chosen to correspond to the errors in the solar data. This would perhaps suggest that there are subtleties in the solar rotation profile that are sensitive to the difference in the averaging kernels. The test 2 profile was based on a previous inversion solution (see Appendix A) and might therefore be smoother than the true solar profile because of the smoothing effect of the original inversion. Alternatively, the cause of the difference between the 2dRLS and 2dSOLA results might be something other than differences in resolution properties between the different methods: for instance it may be that the errors in the real data are not uncorrelated and random, as was assumed for the artificial data. Until the differences are understood, the results obtained at high latitude must evidently be regarded with some caution.

\subsection{Rotation of the Radiative Interior}

Beneath the tachocline, in the radiative interior, the rotation rate appears to vary little, if at all. Its value is around $430 \mathrm{nHz}$. However, there is some evidence, visible both in Figure 3 and in the cuts in Figure 7, for a somewhat localized increase near $r=0.6 R$ at latitude $\simeq 60^{\circ}$. (The fact that this feature persists to higher latitudes in the 2dRLS inversion than it does in others is almost certainly a feature of the extrapolation in regions of little data, mentioned above.) Further observations are obviously required to establish the reality of this feature.

As indicated in Figure 3, we judge that we cannot make reliable inferences below a radius of about $0.5 R$ from just 144 days of data. For inferring the rotation somewhat deeper down, a better data set would seem to be that from the LOWL instrument (Tomczyk et al. 1995b), for which data exist for a longer time series of observations (for inversions see, e.g., Schou, Tomczyk, \& Thompson 1996; Corbard et al. 1997). Inversions from SOI-MDI in this region will improve once observations extending over a longer period have been analyzed and inverted.

For inferring the rotation of the solar core from p-mode frequency splittings, one needs the very lowest degree modes. For these modes long time series and high stability are more important than spatial resolution and very high temporal duty cycle. Currently the best data sets for this 


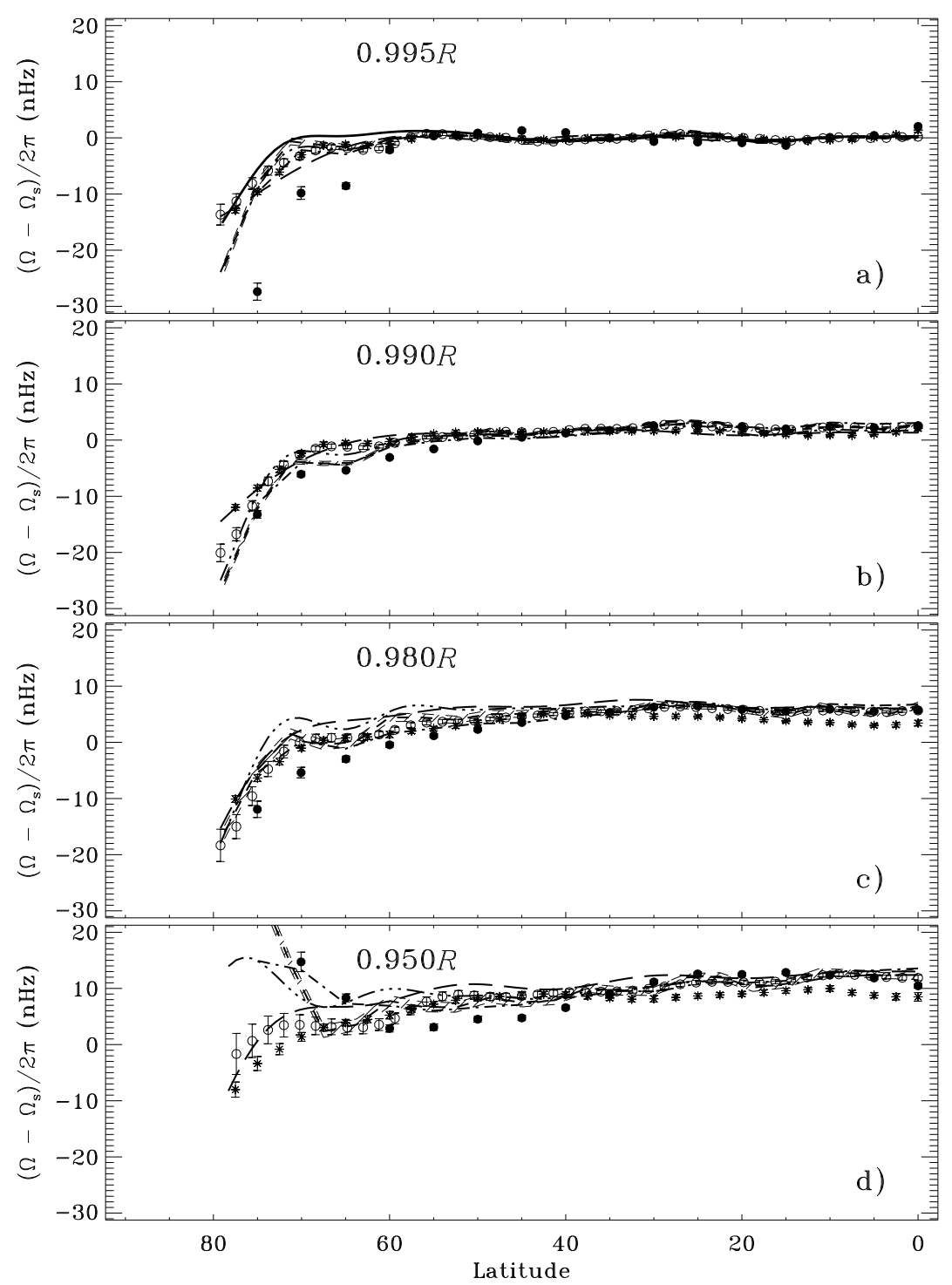

FIG. 10.- Residual $\left(\Omega-\Omega_{s}\right) / 2 \pi$ of the inferred rotation rate at different fixed radii (as labeled), plotted against latitude, after subtraction of fitted three-term rotation rate at $r=0.995 R$ (cf. eq. [17] and Table 2). The heavy solid curve shows the corresponding result obtained by Kosovichev \& Schou (1997) from analysis of f-mode frequencies. Otherwise symbols and line styles are as in Fig. 7. The solution has been restricted to the region outside the shaded area in Fig. 3.

purpose are therefore still ground based, from instruments such as LOWL, BiSON, and IRIS (e.g., Tomczyk et al. 1995b; Elsworth et al. 1994; Lazrek et al. 1996), all of which have observed for several years. Unfortunately, the current inversions based on ground-based data do not agree in the solar core, making evident the need for further observations and the development of better analysis methods.

\section{SUMMARY AND DISCUSSION}

The initial 144 day SOI-MDI data have led to substantially refined helioseismic deductions concerning the solar angular velocity, both in the convection zone and in the transition to the radiative interior. The resolution of the rotational shearing layer just beneath the solar surface and of the tachocline immediately beneath the convection zone has been improved substantially. The data have also revealed that in the convection zone the angular velocity is somewhat lower at very high latitudes than the values obtained by expressing $\Omega$ as the simple three-term expan- sion of equation (17) in colatitude that was originally deduced from the direct surface Doppler measurements of rotational velocity. Furthermore, we have found evidence for a submerged band of fluid near latitudes $75^{\circ}$ rotating more rapidly than its immediate surroundings. We call this phenomenon a submerged polar jet.

We reiterate that our inferences concern only the northsouth symmetric component of $\Omega$; linear rotational splitting of normal modes of global oscillation, from which our inferences are derived, is wholly unaffected by the antisymmetric part. Accordingly, for ease of presentation in our discussion, we often refer to a feature such as the polar jet in the singular. However, the reader should keep in mind that there may be a feature in both hemispheres or in one hemisphere only and that the magnitudes that we quote are actually north-south averages.

The dynamical implications of these features are currently difficult to assess, for the necessary theoretical framework is incomplete. We lack comprehensive theoretical models 


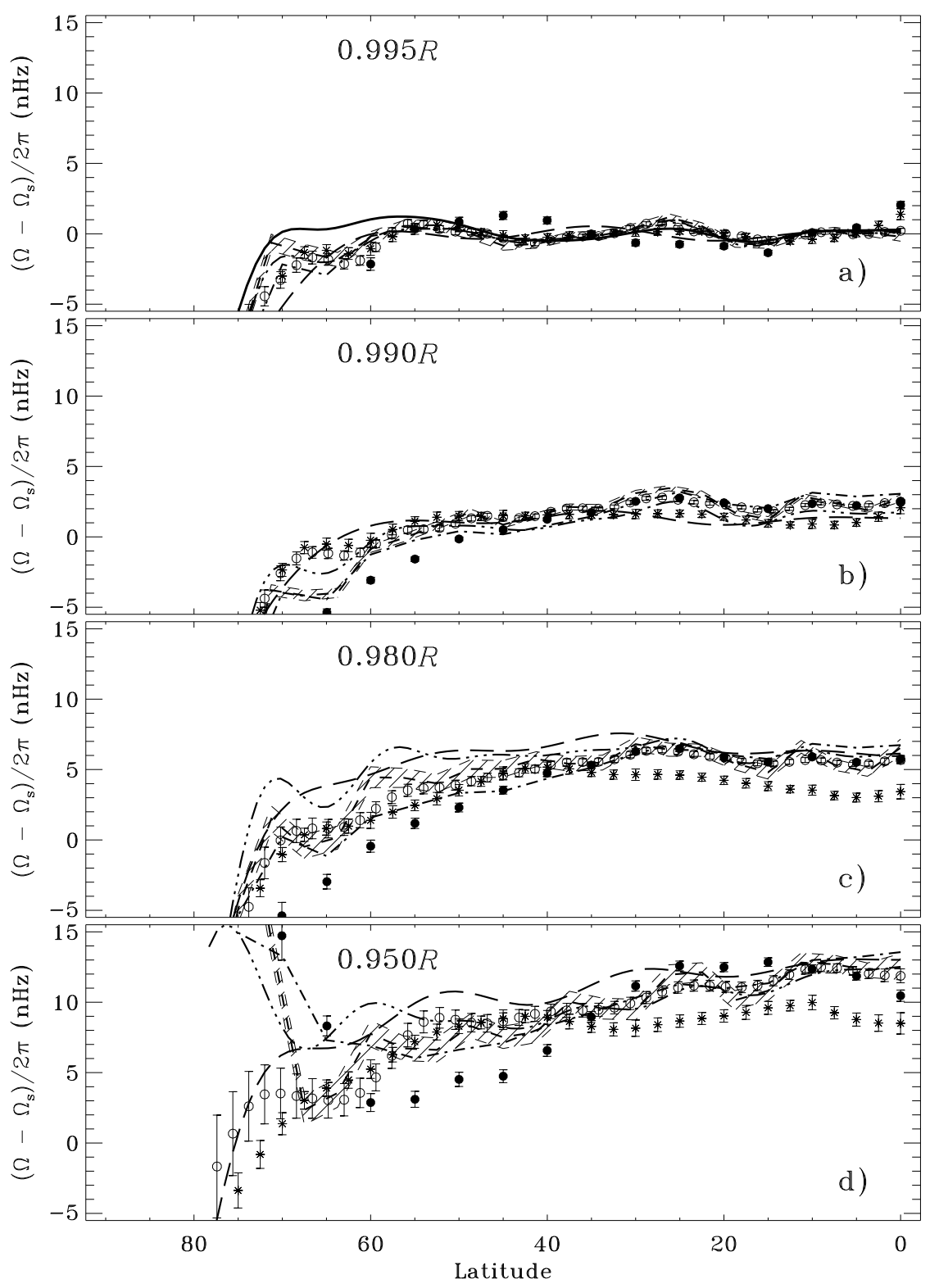

Fig. 11.-As Fig. 10, but over a smaller range in residual to accentuate the spatial fluctuations associated with alternating bands of faster and slower rotation.

that account for the coupling of the highly turbulent convection with rotation. These are needed for developing physical intuition about the operation of various agents that force meridional flow and about the manner in which that flow redistributes angular momentum. Predicting the detailed nature of the mean zonal (east-west) and meridional flows has proved to be very difficult, for to do so reliably requires either numerical simulation of compressible fluids with far more degrees of freedom than are currently tractable on any computer, or a deeper understanding than we have at present of the nature of the smaller scales of turbulence that cannot yet be resolved in full simulations and that must therefore be studied either analytically or by separate simulation.

As discussed in more detail in $\S 1$, the early pioneering simulations of convection in rotating spherical shells (e.g., Gilman 1977, 1979, 1983; Glatzmaier \& Gilman 1982;
Glatzmaier 1985, 1987) did yield differential rotation, but the flows - and in particular the rotation being nearly constant on cylinders - are not in accord with the helioseismic findings. It was quite validly pointed out by Gough et al. (1993) that the then current helioseismic inversions were based on only the low- $a$ coefficients and so had little latitudinal resolution, so that rotation on cylinders within the convection zone could not completely be ruled out by the helioseismic data alone (but see also Schou \& Brown 1994). With the higher $a$-coefficients and consequently better latitudinal resolution of the present SOI-MDI data set, however, we can confidently rule out the rotation-on-cylinders model considered by Gough et al. More recent simulations of compressible convection, studied just in planar layers representing subdomains of a rotating spherical shell in order that the spatial resolution required for representing highly turbulent flow can be attained, have provided further 


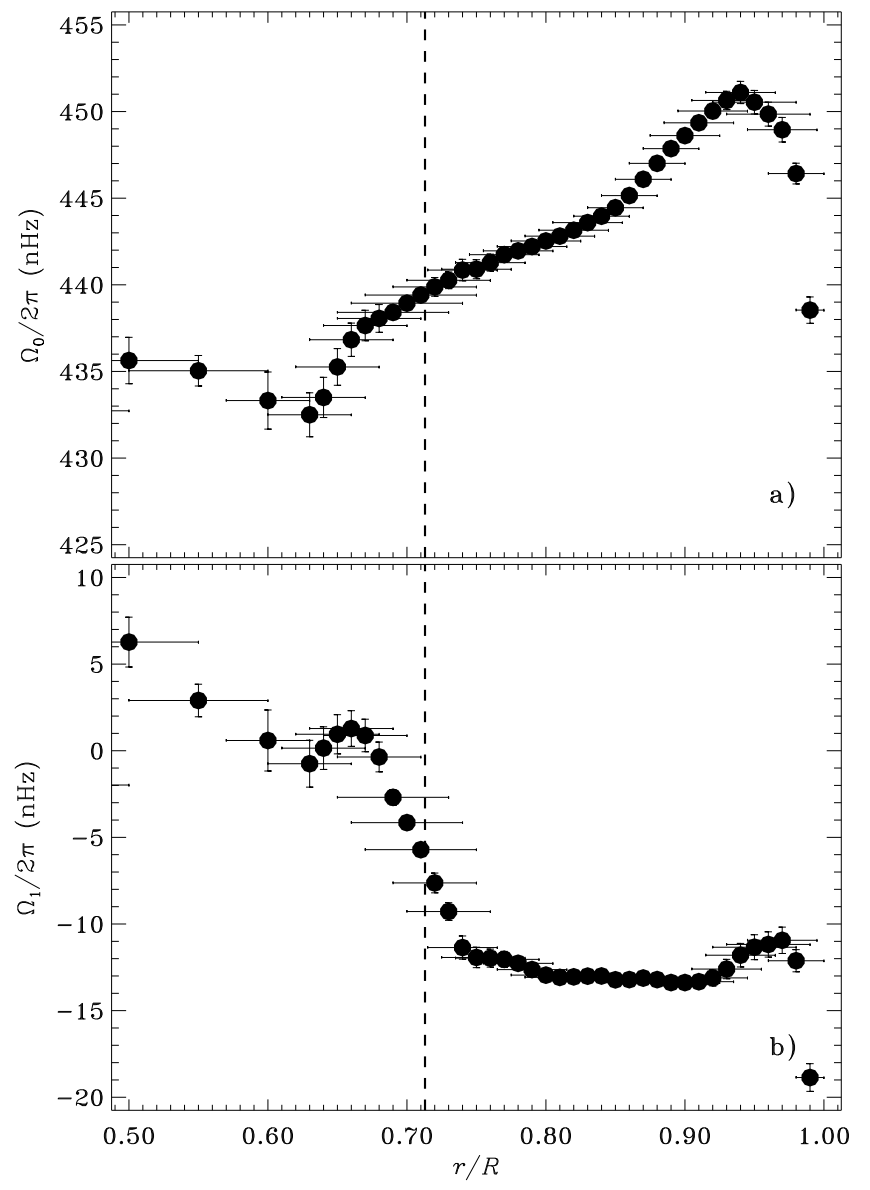

FIG. 12.- Inferred $\Omega_{0}$ and $\Omega_{1}$ in the expansion given by equations (12) and (13) as functions of radius. Horizontal bars denote the FWHM of the $1 \mathrm{~d}$ SOLA averaging kernels used to infer $\Omega_{j}(r)$.

insight into the way by which large-scale zonal and meridional flow can coexist with the intense convection (Pulkkinen et al. 1993; Toomre \& Brummell 1995; Brummell et al. 1996, 1998). The mechanisms responsible for driving the zonal flow are quite different when turbulent rather than laminar states of convection are achieved. The presence of coherent structures superposed on the smaller scale turbulence influences the turbulent Reynolds stresses, which drive a zonal flow with amplitude that is almost independent of depth throughout much of the convecting layer, not unlike that in the Sun. Moreover, they are accompanied by prominent shear layers near the upper and lower boundaries of the convection zone. These angular velocity profiles bear some resemblance to the profile in the Sun. However, detailed comparison must await imminent simulations with the appropriate geometry of a full spherical shell; large-eddy simulations with resolution comparable to that of the recent planar studies, incorporating subgridscale descriptions of turbulence, have recently become feasible on massively parallel computers (e.g., Glatzmaier \& Toomre 1995; Clune et al. 1998).

There are a number of striking features of the variation of the angular velocity in the convection zone. The first is that, except near the pole, the simple three-term expansion of equation (17) captures the dominant dependence on colatitude with remarkable precision, both near the surface and at greater depths (see Table 2). This may provide some clue as to how the overall dynamics of the convection zone operates. At high latitudes, however, the SOI-MDI data reveal (cf. Fig. 10) that the polar regions appear to rotate considerably more slowly than what one would expect from extrapolating the three-term expansion. The anomalous rotation exists poleward of latitude $70^{\circ}$, coinciding approximately with the region from which the fast component of the solar wind emanates along smooth open polar lines of magnetic field. That is suggestive of a causal connection and a possible association with the solar cycle. Accordingly, we suspect that, together with the polar jet, this feature of the rotation may be time dependent. Conflicting inferences regarding time dependence in the rotation profile have been drawn from earlier less well resolved data (Schou 1991a; Goode \& Dziembowski 1991; Gough et al. 1993; Gough \& Stark 1993a, 1993b). It would be very desirable (although difficult because of the foreshortening) to obtain accurate spectroscopic measurements of the surface rotation rate near the pole.

The second feature to which we draw attention is that superposed on the relatively smooth $\Omega$ profile described by the three-term expansion there are alternating bands of slightly faster and slower rotation, each extending some $10^{\circ}-15^{\circ}$ in latitude, with peak velocity amplitudes of about $5 \mathrm{~m} \mathrm{~s}^{-1}$. This is to be compared with the much faster primary velocity of nearly $2000 \mathrm{~m} \mathrm{~s}^{-1}$ at the equator. The weak banded flow may be followed in the inversions to a depth of perhaps as much as $0.05 R$ in Figure 11 and appears to coincide with the so-called torsional oscillations that have been observed directly in Doppler measurements of the rotation of the photosphere, most recently from GONG (e.g., Howard \& LaBonte 1980; Snodgrass, Howard, \& Webster 1985; Ulrich et al. 1988; Hathaway et al. 1996). From those studies it is reported that the pattern of bands propagates equatorward from latitudes of at least $70^{\circ}$ in about $20 \mathrm{yr}$, the oscillation period at any fixed latitude being about $22 \mathrm{yr}$.

By comparing inversions of SOI-MDI data from five adjacent 72 day intervals, the equatorward propagation is evident, at a rate which is not inconsistent with the longer term surface Doppler measurements (Schou et al. 1998). Whether or not the phenomenon persists poleward of $70^{\circ}$, where Doppler measurements have not been possible and where, as we have discussed already, the mean angular velocity is anomalously low, is yet to be ascertained.

The relative feebleness of the torsional flow in the Sun is perhaps surprising, given the prominence of the alternating bands of fast and slow flow in the giant gaseous planets. It has been suggested that on Jupiter the banded flow might be primarily a response of the stably stratified atmosphere to jostling by turbulent convection below and that the differential rotation deeper in the planet might be different (e.g., Ingersoll 1990; Cho \& Polvani 1996). Yet the Sun has a stably stratified atmosphere above a turbulent layer too. There are, however, some differences. The solar measurements are made at the top of the convection zone, which in Jupiter is partly shrouded from view. Moreover, on Jupiter there is no indication of equatorward propagation of the zonal pattern.

In contrast with the smooth variation with latitude, the change in $\Omega$ with radius is in some places quite abrupt: specifically, immediately beneath the base of the convection zone and in the shear layer just below the solar photo- 
sphere. The upper shear layer is shown in selected cuts at constant latitude in Figures 7 and 8. At low latitudes there is a prominent rise in $\Omega$ with depth immediately beneath the surface, peaking at about $r=0.93 R-0.95 R$. Such behavior may be related to the observed low-latitude rapid rotation of both sunspots and supergranulation patterns relative to the photospheric gas (e.g., Wilcox \& Howard 1970; Duvall 1980; Snodgrass 1983; Howard, Gilman, \& Gilman 1984; Nesme-Ribes, Ferreira, \& Mein 1993). The difference of about $3 \%$ in the rotation rates has led to speculation that the sunspots and the supergranulation are sensing somewhat more rapid rotation at the depth to which they extend. Some attempts to explain the angular velocity gradient have invoked angular momentum conservation in vertically moving convecting fluid (e.g., Foukal \& Jokipp 1975; Foukal 1977; Gilman \& Foukal 1979). However, angular momentum cannot be conserved exactly, because if it were, the angular velocity at a depth of $0.07 R$ would exceed that at the surface by $14 \%$, whereas only a $4 \%$ increase is inferred from the inversions. Perhaps the tendency to conserve angular momentum is influencing a much more complicated nonaxisymmetric flow, which is itself driven by nonaxisymmetric stresses. Whatever is occurring, so simple a statement does not alone explain why the rise in $\Omega$ with depth stops where it does and why the gradient then reverses sign.

At higher latitudes, such as near $60^{\circ}$, the sign of the radial shear near the surface is reversed: $\Omega$ initially decreases with depth, attaining a minimum at about $r=0.99 R$, beneath which is a gradual yet nonmonotonic rise. The dynamics in the region is evidently quite complicated, and the flow is possibly unsteady on scales large compared with that of the dominant eddies. It will be interesting to determine whether in the future the details of this behavior will have changed.

A further notable feature at high latitudes is the localized domain of relatively rapid rotation evident at a radius of about $0.95 R$ in the $75^{\circ}$ cut in Figure 8. This jetlike flow has not been detected previously from other helioseismic data, and even from the SOI-MDI data its detection is tentative. It will be interesting to learn from future data whether or not this feature persists too. We should keep in mind that determining the frequency splitting of the global modes requires lengthy averaging in time, which tends to mask temporal variation in $\Omega$. Indeed, it is highly likely that the polar jet is also nonaxisymmetric, perhaps with undulating streamlines not unlike those of terrestrial jetstreams. One must appreciate, however, that the detailed dynamics of such a solar jet in a convectively unstable region cannot be identical to the terrestrial jetstream, which exists in a layer of the atmosphere that is very stably stratified.

The layer of shear in angular velocity near the base of the convection zone is a transition from the latitudinally varying rotation of the convection zone to almost uniform rotation of at least the outer layers of the radiative interior. Some possible aspects of the dynamics of this layer have been considered by Spiegel \& Zahn (1992). They separated the genuine convection zone, in which it was assumed that there is no radial shear, from the stably stratified shear layer beneath, which they called the tachocline. It is evident from the inversions illustrated in Figure 7 that the Reynolds stresses in the convection zone are either not strong enough to resist the local torque applied by the radiative interior or are not of the appropriate nature to maintain radially invariant rotation, for the shear in $\Omega$ extends into the con- vection zone. Nevertheless, we follow Spiegel \& Zahn (1992) and reserve the term tachocline for only that portion of the shear layer that is located in the convectively stable radiative zone, where we believe the dynamics to be quite different from that in the convection zone.

There is currently no general agreement on the processes that dominate the tachocline dynamics. Spiegel \& Zahn (1992), and subsequently Elliott (1997), have assumed the presence of horizontally isotropic shear-generated twodimensional turbulence, which acts as a viscosity in such a way as to force the fluid toward uniform angular velocity on spherical surfaces. The angular velocity deep in the radiative interior is determined in the steady state by the condition that there is no net torque across the tachocline, although a convincing explanation of how that state might be achieved by purely hydrodynamic means in the face of global spin-down is lacking. Moreover, there is evidence from extensive theoretical and observational studies of the stably stratified terrestrial stratosphere that layerwise twodimensional turbulence does not lead to uniform rotation (McIntyre 1994). It is therefore likely that the rigidity of the interior can be provided only by a large-scale magnetic field (cf. Mestel \& Weiss 1987; Gough \& McIntyre 1998) and that the tachocline provides an interface in which radial field lines might connect the convection zone with the radiative interior only near the latitudes at which there is essentially no radial shear. The dynamics has not yet been modeled in detail and is evidently made complicated by the uncertain degree of convective overshooting into the stably stratified medium below.

Figure 7 shows cuts of $\Omega$ at constant latitude. Those at the higher latitudes, $60^{\circ}$ and $75^{\circ}$, may appear to suggest that the shear layer is distinctly broader near the poles than it is at the equator. It is perhaps not surprising that this is so, partly because the resistance to vortex stretching in the nearly isentropic convection zone transmits the interior angular velocity further near the poles, where the vorticity is vertical, than near the equator, where it is horizontal. Moreover, if the radiative interior is held rigid by a magnetic field (we have no alternative plausible suggestion to offer), that field is likely to have been swept horizontally away from the poles by the circulation in the tachocline and is therefore expected to be weakest near the poles. It should be borne in mind, however, that the vorticity generated in the largescale convective eddies is comparable to that associated with the rotation. Consequently, vortex stretching is not resisted in practice to the degree that one would expect in a purely isentropic fluid. We therefore recognize that we cannot yet distinguish between that part of the radial variation of $\Omega$ that is transmitted from the tachocline and that part that is intrinsic to the convective motion. Finally, we must point out that the averaging kernels obtained from the inversions have radial extents of about $0.1 R$, often with sidelobes near the photosphere, so we cannot rule out a much more abrupt transition than those suggested by the figures or that the apparent latitude dependence is caused by the limited resolution. It is important to devote further effort to studying this issue, because the properties of the tachocline are of fundamental dynamical importance. Moreover, the tachocline is likely to be a site of magnetic enhancement and may be at least partially responsible for controlling the cycles of solar activity (e.g., Spiegel \& Weiss 1980; Rosner 1980; Glatzmaier 1985; Weiss 1994).

At this stage the helioseismic observations and their 
interpretations are considerably in advance of predictive dynamical theories. Given the complexity of the physics, that situation will persist for possibly quite some time. It is to be hoped that in the near future some simulations of turbulent convection coupled to rotation may begin to provide plausible explanations for what may be happening in the solar convection zone and that they may also yield a perspective of other types of mean flow and differential rotation profiles that could be achieved in such systems.

We have high hopes that the greater accuracy of the frequency splitting that will be achieved when more data have been accumulated will improve the resolution of the variation of $\Omega$ to the extent that greater dynamical insight will soon be achieved. Improved precision of the lowest degree modes is especially needed, for then we shall be able to probe the rotation of the core. The indications from the BiSON and LOWL data (Elsworth et al. 1995; Tomczyk, Schou, \& Thompson 1995; Chaplin et al. 1998) that the core is rotating slowly are particularly intriguing. If the core really does rotate slowly, it is likely that there has been some angular momentum draining process, perhaps by Lorentz forces or perhaps by wave transport. However, the low splitting of the low-degree modes has been challenged (e.g., Lazrek et al. 1996), so the matter is far from having been settled. It is extremely important, therefore, to devote considerable effort to understanding the statistics of the modes in order to obtain unbiased estimates of the splitting. It is also essential to our understanding of the dynamics of the Sun to learn how the angular velocity varies with time and to what extent those variations are associated with the solar cycle. That will require many years of data. We trust that with these in hand we shall improve our insight into the global dynamics, which can be applied not only to the Sun, but also to the rotation of other stars.

The authors acknowledge many years of effort by the engineering and support staff of the SOI-MDI development team at the Lockheed Palo Alto Research Laboratory (now Lockheed Martin) and the SOI-MDI development team at Stanford University. The SOI-MDI project is supported by NASA grant NAG 5-3077 to Stanford University, with subcontracts to Lockheed Martin, the University of Colorado, and Harvard University. SOHO is a mission of international cooperation between ESA and NASA. D. A. Haber and $\mathbf{J}$. Toomre are also partially supported by NASA through grant NAG 5-2256 and by the NSF through grants ECS-9217394 and AST 94-17337. NOAO is operated by AURA under cooperative agreement with NSF. D. O. Gough, T. Sekii, R. Howe, and M. J. Thompson are supported by the UK Particle Physics and Astronomy Research Council, through grants GR/K46767 and GR/ J00588. S. Basu, J. Christensen-Dalsgaard, and F. P. Pijpers were partially supported by the Danish National Research Foundation through the establishment of the Theoretical Astrophysics Center. We thank S. Tomczyk and P. R. Wilson for helpful comments on an earlier version of the manuscript.

\section{APPENDIX A}

\section{HARE AND HOUNDS}

As a controlled experiment to test the sensitivity and reliability of our procedures, and the reliability of the interpretations we place on the inversions, we have conducted a hare-and-hounds exercise. One of us (A. G. K.), in the role of hare, invented two fictitious rotation profiles, not dissimilar to the rotation profiles that we are deducing for the solar interior. The two artificial profiles, test1 and test2, are illustrated in Figure 13. Using a standard model of the Sun's spherically symmetric hydrostatic structure to construct the kernels $K_{n l m}$, the hare computed frequency splittings for each of test1 and test2 according to equation (1). He then fitted splitting coefficients to the splittings, in the manner of equation (2), corresponding to the range of $(n, l)$ multiplets and numbers of coefficients available from the real SOI-MDI data. To these artificial splittingcoefficient data he also added independent, Gaussian-distributed errors with zero mean and standard deviations equal to the estimated standard deviations of the SOI-MDI data errors.

The artificial data were then distributed to six participating packs of hounds, who were asked without knowledge of what the test 1 and test 2 looked like to use the data to deduce what they could about them. The hounds were told the data values, the values of $n, l$, and $j$ to which they corresponded, and the associated standard deviation of the noise that had been added to each datum. They were told nothing else about the rotation profiles, nor were they given the spherically symmetric model or the kernels $K_{n l m}$ or $K_{n l s}^{(a)}$.

The participating hound packs used different inversion methods and initially worked independently of one another. The packs were H. M. A., S. B., and S. M. C. using $1.5 \mathrm{dRLS}$; J. C.-D. using $2 \mathrm{dSOLA}$; A. E.-D. and S. G. K. using OMD and 1.5dSOLA; R. H. and M. J. T. using $2 \mathrm{dRLS}$; F. P. P. using $1 \mathrm{~d} \times 1 \mathrm{dSOLA}$; and T. S. using $1 \mathrm{~d} \times 1 \mathrm{dOLA}$. The hounds initially reached their own conclusions about test 1 and test 2 on the basis of their inversions, without knowing what any of the other packs had found. This was the first stage of the hare-and-hounds exercise. Then, as a second stage, the hounds discussed what they had found and reached a set of joint conclusions summarizing the deductions about the two test profiles that they considered most reliable.

We present some results of the hare-and-hounds exercise in the same format as our solar results. Figures 14, 15, 16, and 17 for test 1 and Figures 18, 19, 20, and 21 for test 2 correspond to Figures 3, 7, 9, and 11. There was generally very good agreement among the different packs of hounds regarding the principal features of test 1 and test 2 . For that reason we shall not report in detail here on the separate conclusions drawn by the individual packs. A fuller report on the exercise will be presented elsewhere (Kosovichev et al. 1998). Some of the results of the individual inversions can, however, be seen in Figures 14 and 15 for test 1 and in Figures 18 and 19 for test 2.

The joint conclusions of the hounds, briefly summarized, were as follows: For test1, the hounds correctly inferred that the 

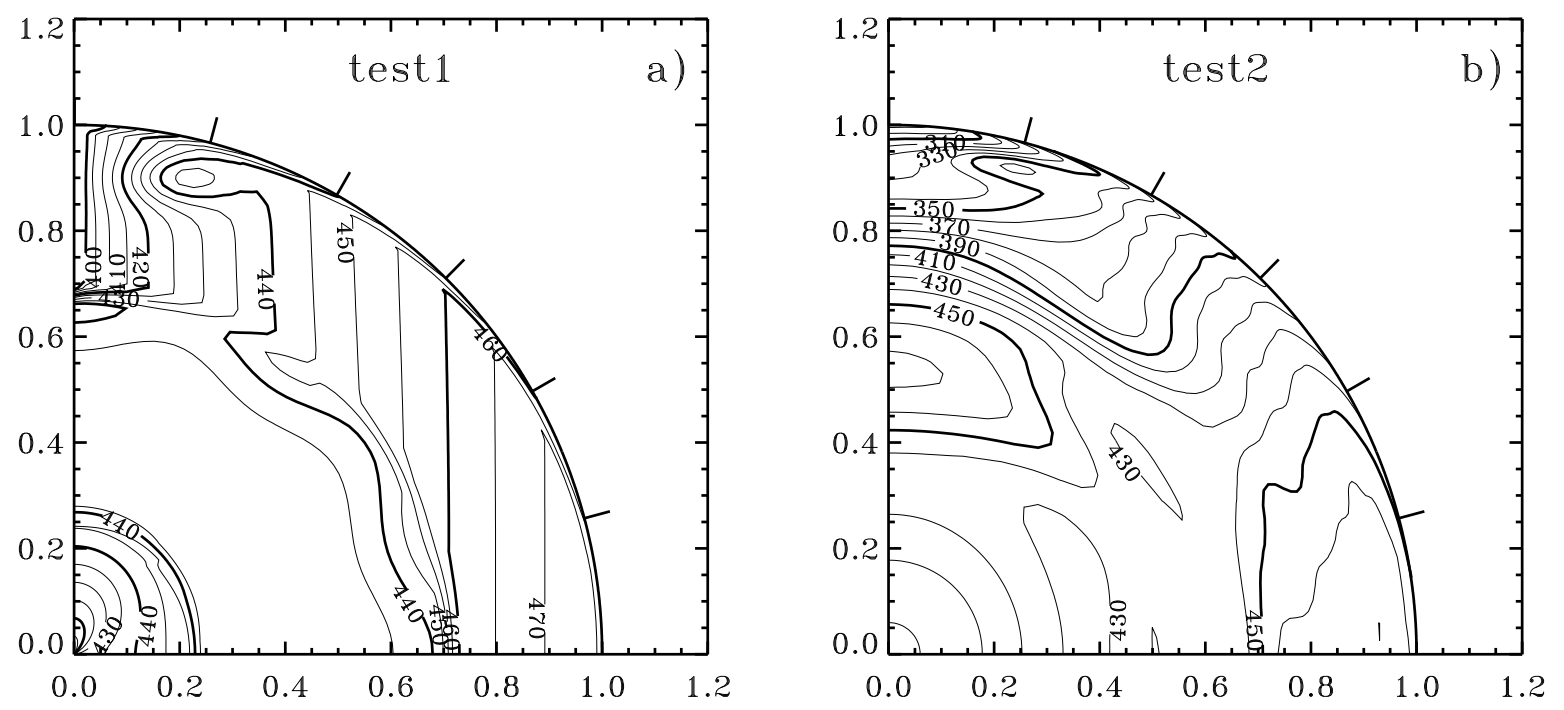

Fig. 13.-Two artificial rotation laws set up by the hare. Panel $a$ shows the test1 rotation profile $\Omega / 2 \pi$ used in the hare-and-hounds exercise, while panel $b$ shows the test 2 profile. Contours at constant rotation rate are shown, the levels being separated by $5 \mathrm{nHz}$ in panel $a$ and $10 \mathrm{nHz}$ in panel $b$. For clarity, selected contours are shown as bold. In both cases, the highest values of the rotation are in the near-surface equatorial regions. Tick marks at the edge of the outer circle are at latitudes $15^{\circ}, 30^{\circ}, 45^{\circ}, 60^{\circ}$, and $75^{\circ}$.
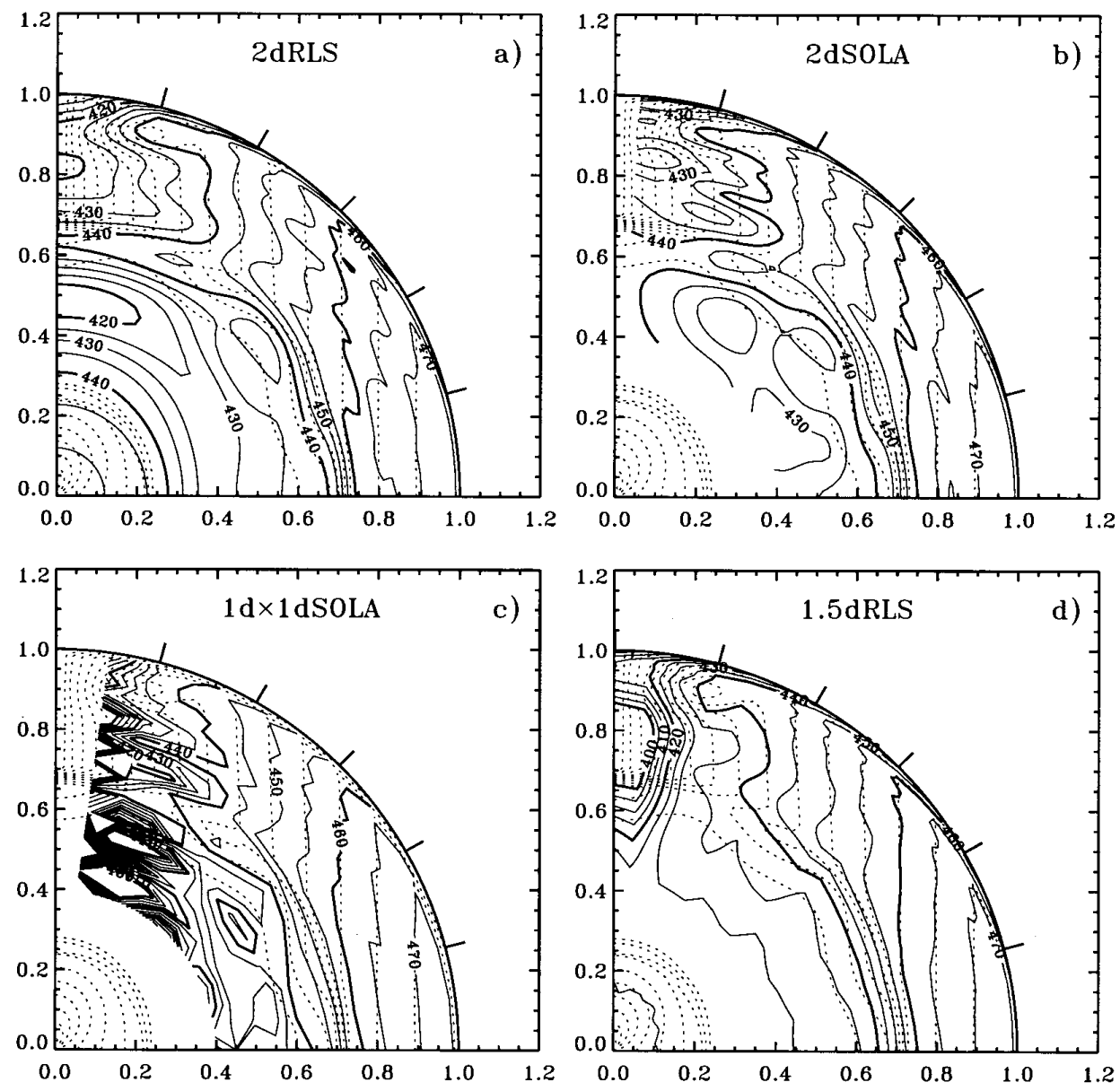

FIG. 14. Contour plots of solutions for the rotation rate $\Omega / 2 \pi$ against radius and latitude for the same four methods as illustrated in Fig. 3, compared with the assumed rotation law, for test1: (a) 2dRLS; (b) 2dSOLA; (c) 1d $\times 1 \mathrm{dSOLA} ;(d) 1.5 \mathrm{dRLS}$. The corresponding contours for the actual test1 rotation profile are shown (dotted lines). See also caption to Fig. 13. 


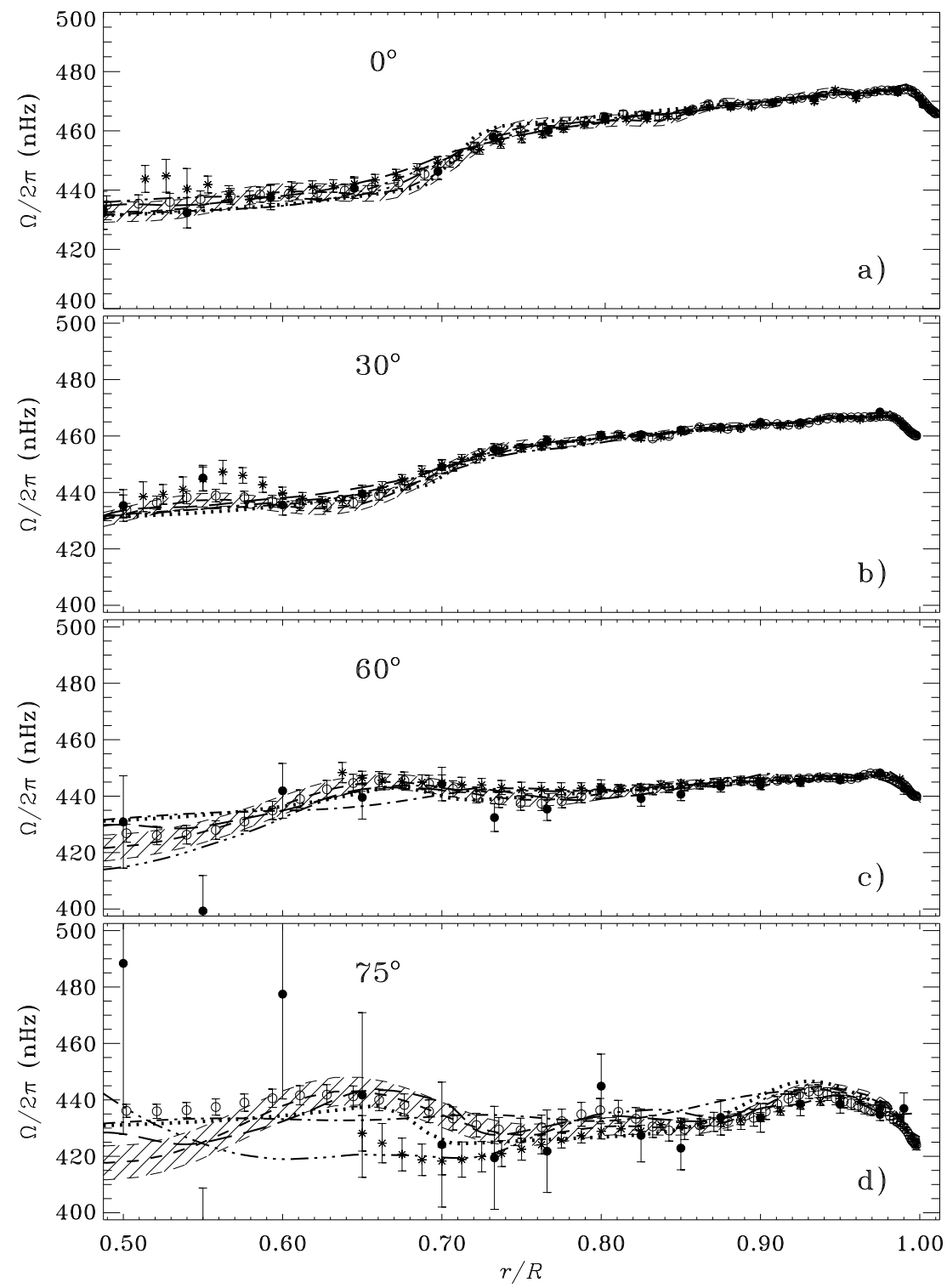

Fig. 15.- Radial dependence of $\Omega / 2 \pi$ at constant latitude for the solutions for the test 1 case. The actual test 1 rotation is shown with the dotted line. Line and symbol styles are 2dRLS (dashed line with error band), 1.5dRLS (dot-dashed line), OMD (triple-dot-dashed) 2dSOLA (open circles with $1 \sigma$ error bars), $1 \mathrm{~d} \times 1 \mathrm{dOLA}$ (stars with error bars), $1 \mathrm{~d} \times 1 \mathrm{dSOLA}$ (closed circles with error bars), and $1.5 \mathrm{dSOLA}$ (long-dashed line). Solutions are shown at the following latitudes: $(a) 0^{\circ} ;(b) 30^{\circ} ;(c) 60^{\circ} ;(d) 75^{\circ}$.
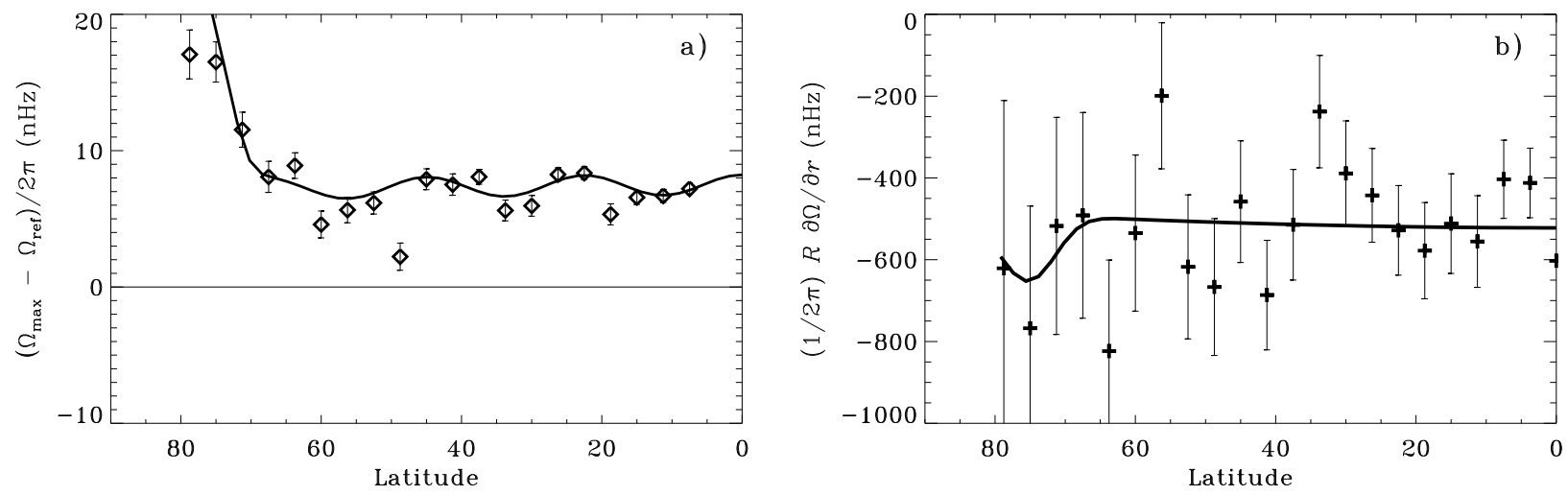

FIG. 16.- Near-surface maximum and derivative in inferred 2dRLS solution for the test1 artificial data (symbols), compared with the "truth" (lines) (cf. Fig. 9). (a) The value at the maximum, relative to the reference value at $0.995 R$. (b) Estimate of the derivative near $0.995 R$. 


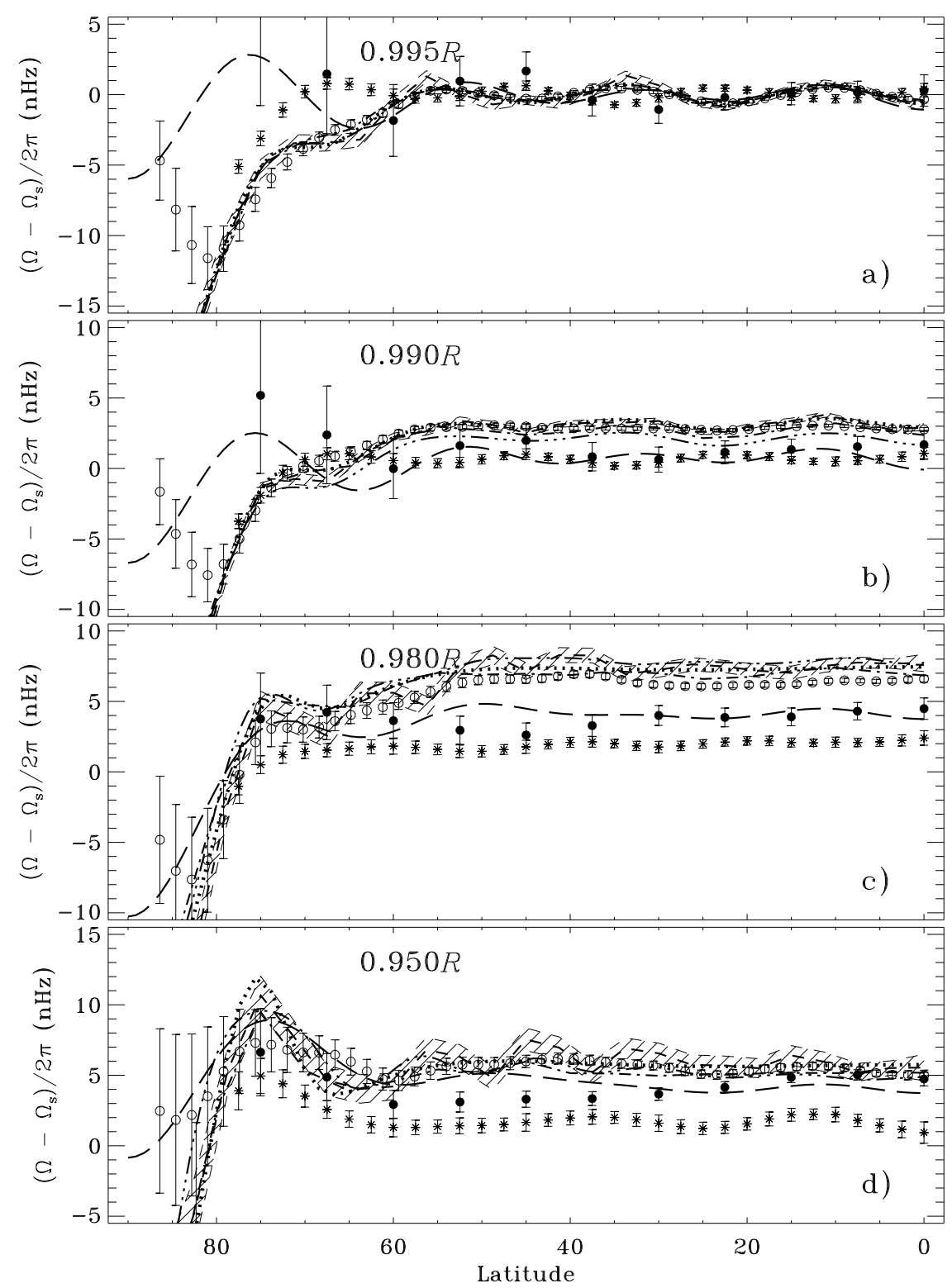

FIG. 17. - Latitudinal dependence of the rotation rate at different fixed radii (as labeled), after subtraction of fitted three-term rotation rate at $r=0.995 R$, for the test 1 case. The line and symbol styles are as in Fig. 15.

rotation was essentially constant on cylinders in the convection zone, at least in the region that is more than $0.4 R$ from the rotation axis. This is seen clearly in Figure 14. It was very striking in the 1.5dRLS inversion (panel d). At smaller distances from the rotation axis (i.e., at high latitudes) the pattern was less clear. The hounds also inferred that the sharp transition in the solar rotation rate between the convective and radiative zones was in the range $0.70 R-0.71 R$ and that it extended from the equator up to a latitude of about $50^{\circ}$. They also found a surface shear layer, with the maximum rotation rate (about $10 \mathrm{nHz}$ faster than the surface rate) occurring at about $0.98 R$ (see Fig. 15). That shear layer was found to continue up to very high latitudes. Below the shear layer, the rotation rate was seen to fall slowly with depth. These qualitative inferences are in good agreement with the properties of the actual test1 profile. Quantitatively, the rotation rate at $0.98 R$ at the equator was estimated to be around $470-475 \mathrm{nHz}$, falling to about $448 \mathrm{nHz}$ at $60^{\circ}$ latitude. For comparison, the actual corresponding test 1 values are 475.5 and $448 \mathrm{nHz}$. Beneath the convection zone, the rotation rate was generally found to be consistent with solid-body rotation at about $430 \mathrm{nHz}$. Furthermore, most of the inversions recovered the weak gradient in the radiative interior present in the model, at least at the equator (cf. Fig. 15). None of the methods provided results on the features in the core.

The hounds also commented, with less unanimity, on a number of small-scale features. The only one to command a wide degree of consensus was a bump in the rotation profile at about $0.93 R-0.95 R$ at around $70^{\circ}-80^{\circ}$ latitude. This was commented on by four of the six packs of hounds. As can be seen from Figure 14, this does indeed correspond to a real feature in the test1 profile.

For test2, the hounds agreed that this looked very similar to a real solar inversion. This was indeed the case; the test 2 model was obtained by linearly interpolating the $2 \mathrm{dRLS}$ inversion results with no modification. Again, the hounds correctly detected 

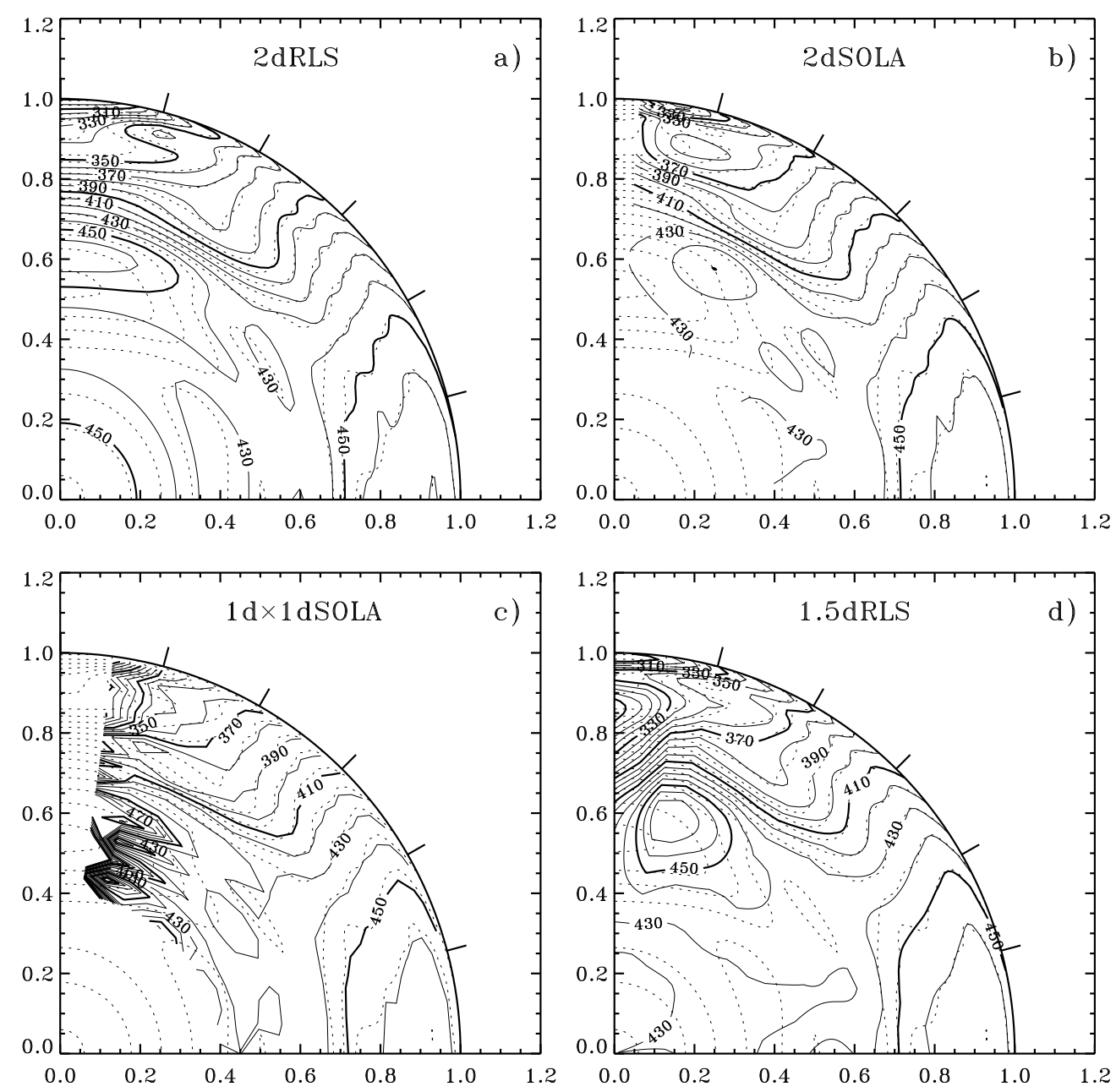

FIG. 18. - Contour plots of solutions for rotation rate $\Omega / 2 \pi$ for the same four methods as illustrated in Fig. 3, compared with the assumed rotation law, for test2: (a) 2dRLS; (b) 2dSOLA; (c) $1 \mathrm{~d} \times 1 \mathrm{dSOLA} ;(d) 1.5 \mathrm{dRLS}$. The corresponding contours for the actual test 2 rotation profile are shown (dotted line). See also caption to Fig. 13.

the near-surface shear layer. They claimed that the sign of $\partial \Omega / \partial r$ in the near-surface layers changed at around a latitude of $55^{\circ}$, which is in fact a feature of the actual test2 rotation law (cf. Fig. 13; see also Fig. 20). Some evidence was found for a low rotation rate near the pole (less than $300 \mathrm{nHz}$ ), which is in fact the case. The location of the tachocline was inferred to be at about $0.71 R$. And once again the rotation rate below the convection zone was found to be consistent with solid-body rotation at $430 \mathrm{nHz}$. The 2dRLS successfully detected the high-latitude jet, a feature that the inverters noted. It was perhaps unfortunate for the purposes of an unbiased comparison that an RLS solution was used as the test 2 profile. This tempers somewhat the apparent success of 2dRLS.

All of the preceding conclusions were based on the blind hare-and-hounds exercise. We have subsequently compared the inferred and original rotation profiles in more detail. In particular, Figures 16 and 20 illustrate the nature of the near-surface shear layer and are to be compared with the corresponding figure (Fig. 9) for solar data. For test2 the variation with latitude of the shear-layer properties is recovered quite satisfactorily except at very high latitude. By contrast, the weaker radial gradient of rotation for test (Fig. 16b) is recovered very noisily. The amplitude of this gradient in the Sun appears to be more similar in magnitude to test 2 than to test1, so that we may hope that in the solar case these properties are reasonably well recovered by the inversions.

Residuals from subtracting fits to the inferred surface rotation are shown in Figures 17 and 21 (to be compared with Fig. 11) for the solar case. In panels $a$ and $b$, real small-scale latitudinal variations in the exact profiles are recovered quite successfully by the inversions, which gives us confidence in our inferences about the so-called solar torsional oscillations. However, the 2dRLS solution in particular also exhibits spurious latitudinal oscillations at greater depths (panels $c$ and d) in the hare-andhounds results, which leads us to be cautious about believing that similar oscillations in Figures $11 \mathrm{c}$ and $11 d$ are real.

What final conclusions can be drawn from the hare-and-hounds exercise? It is very encouraging that for data sets with coverage similar to the SOI-MDI set, the conclusions drawn by the inverters proved to be as reliable as the inverters believed them to be. Thus the inverters were correct in believing that the inversions were reliable in a substantial region where the averaging kernels were successfully localized close to their target locations, i.e., in the near-surface layers, at latitudes up to about $75^{\circ}$, and down to a little beneath the convection zone. We were also encouraged with the degree of agreement between the methods. 


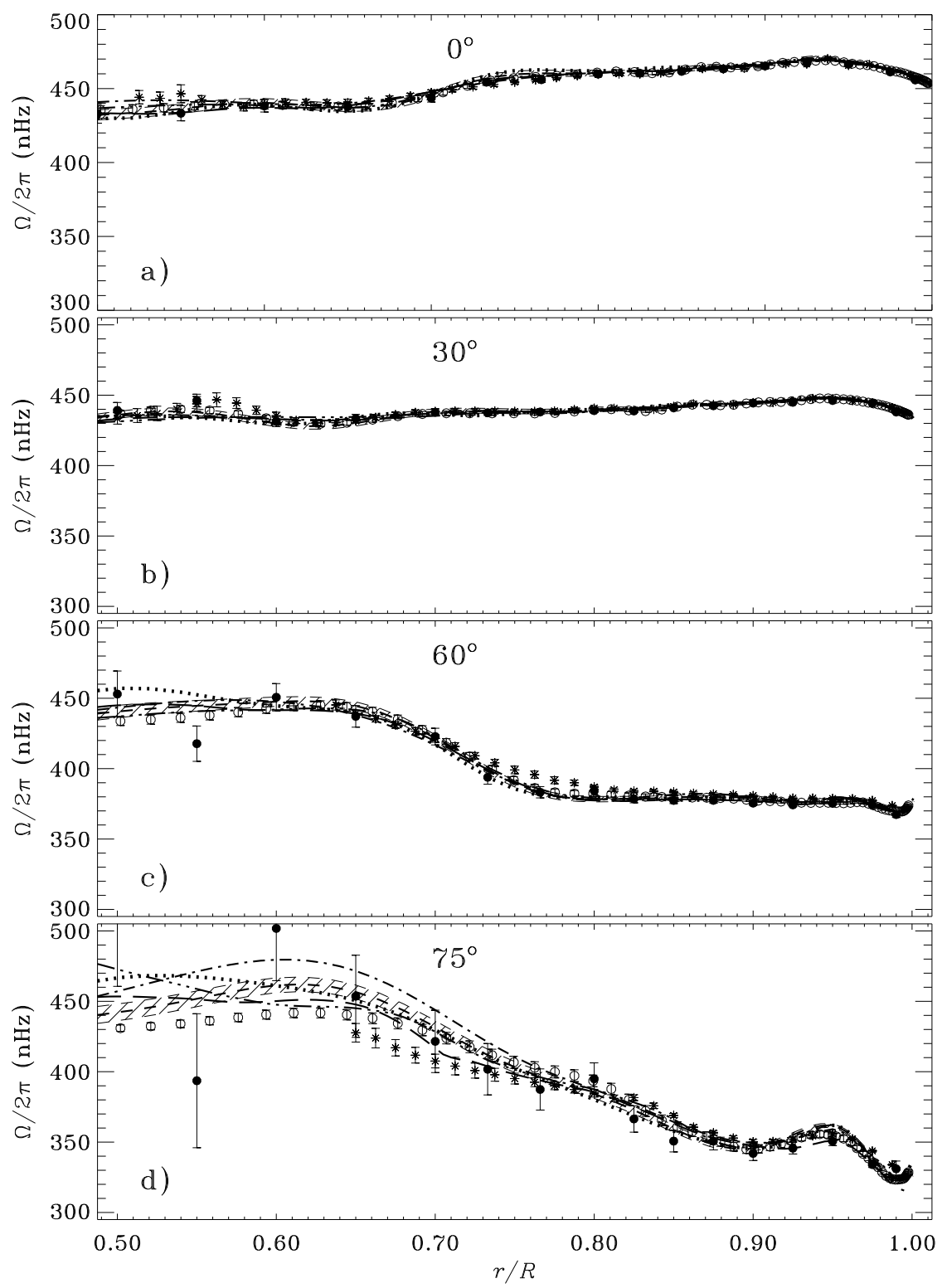

FIG. 19. - Radial dependence of $\Omega / 2 \pi$ at constant latitudes (labeled) for the solutions for the test 2 case. The actual test 2 rotation is shown with the dotted line. The line and symbol styles are as in Fig. 15.
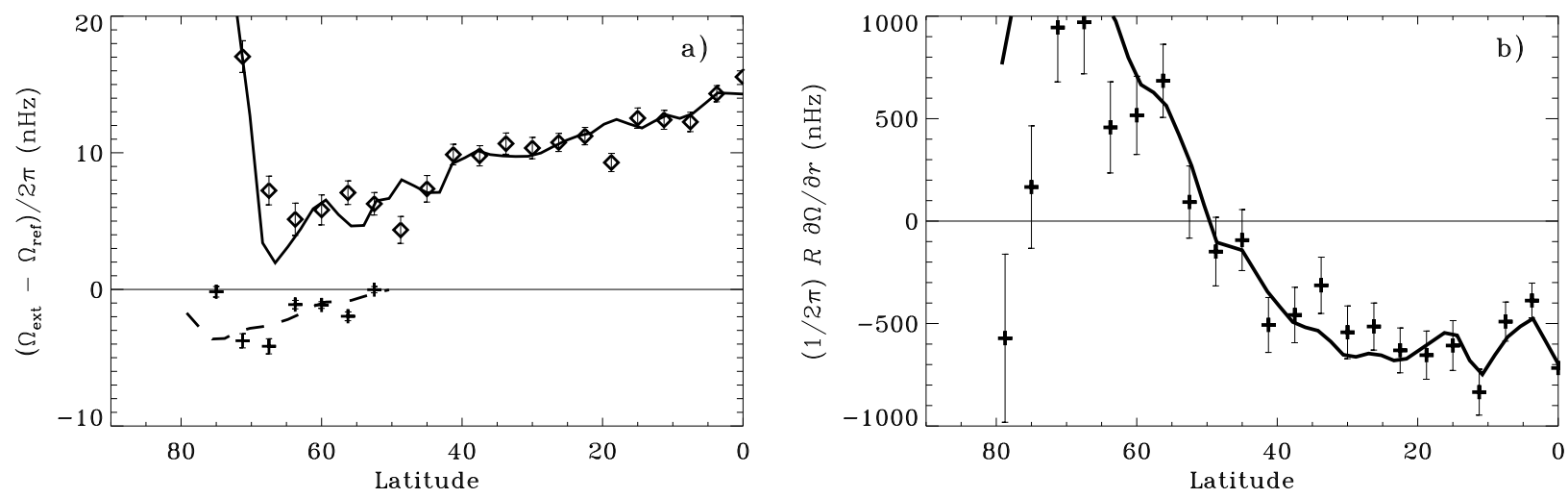

FIG. 20.-Near-surface extrema and derivative in inferred 2dRLS solution for the test2 artificial data (symbols), compared with the "truth" (lines) (cf. Fig. 9). (a) The value at the maximum around $0.95 R$ (diamonds) and the minimum around $0.99 R$ (crosses, for latitude above $50^{\circ}$ ), relative to the reference value at $0.995 R$. (b) Estimate of the derivative near $0.995 R$. 


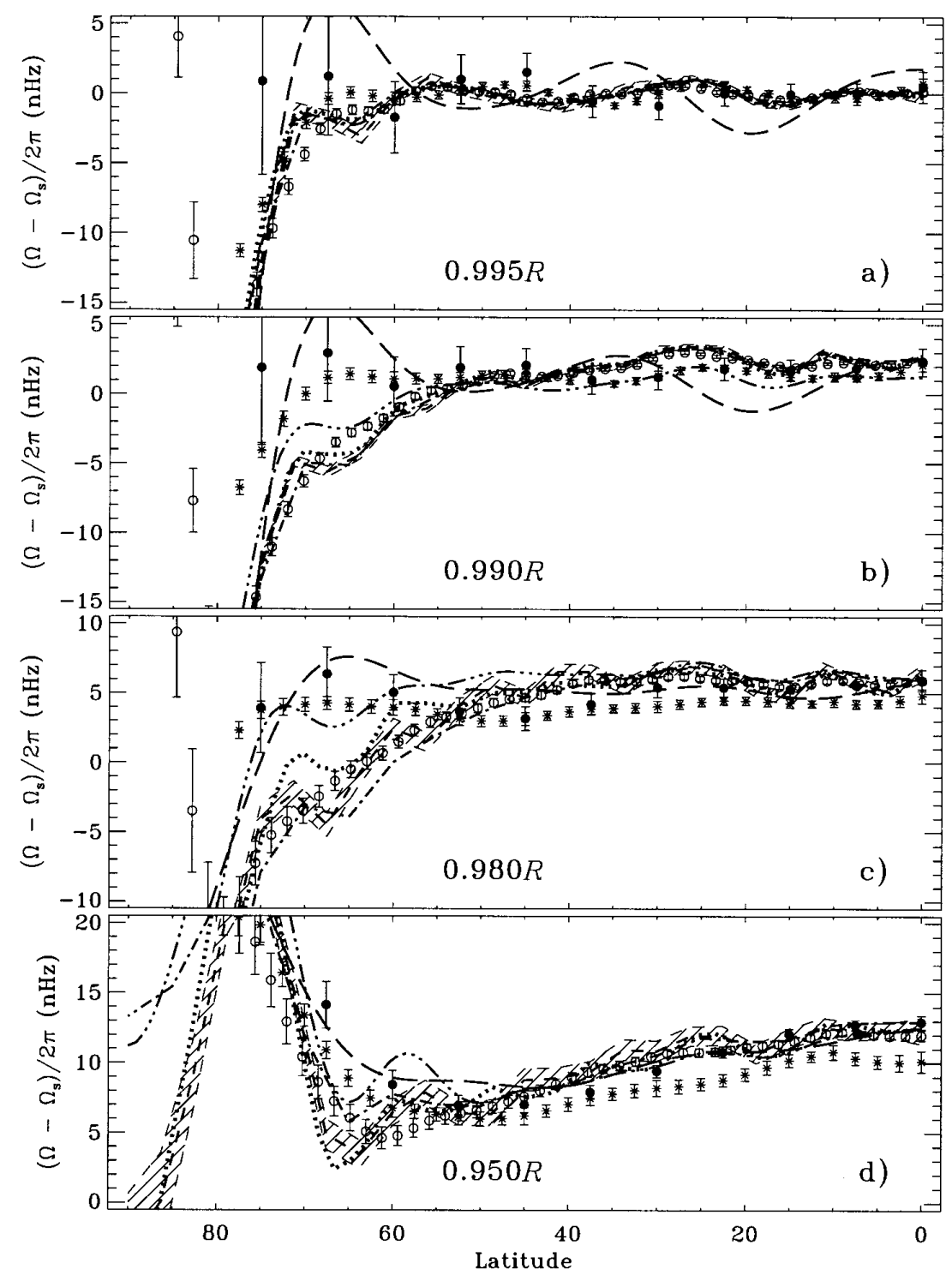

FIG. 21.-Latitudinal dependence of rotation rate at different fixed radii (as labeled), after subtraction of fitted three-term rotation rate at $r=0.995 R$, for the test 2 case. The line and symbol styles are as in Fig. 15.

Antia, H. M., Chitre, S. M., \& Thompson, M. J. 1996, A\&A, 308, 656 Backus, G. E., \& Gilbert, F. 1968, Geophys. J. RAS, 16, 169 Basu, S. 1997, MNRAS, 288, 572

Brown, T. M., Christensen-Dalsgaard, J., Dziembowski, W. A., Goode, P., Gough, D. O., \& Morrow, C. A. 1989, ApJ, 343, 526

Brummell, N. H., Cattaneo, F., \& Toomre, J. 1995, Science, 269, 1370 Brummell, N. H., Hurlburt, N. E., \& Toomre, J. 1996, ApJ, 473, 494 . 1998, ApJ, 493, 955

Cattaneo, F. 1993, in Solar Magnetic Fields, ed. M. Schüssler \& W. Schmidt (Cambridge: Cambridge Univ. Press), 261

Chaboyer, B., Demarque, P., \& Pinsonneault, M. H. 1995, ApJ, 441, 865

Chaplin, W. J., et al. 1998, in Proc. IAU Symp. 181, Sounding Solar and Stellar Interiors (poster volume), ed. J. Provost \& F.-X. Schmider (Nice: Obs. Nice), 731

Charbonneau, P. Christensen-Dalsgaard, J., Henning, J., Schou, J., Thompson, M. J., \& Tomczyk, S. 1998, in Proc. IAU Symp. 181, Sounding Solar and Stellar Interiors (poster volume), ed. J. Provost \& F.-X. Schmider (Nice: Obs. Nice), 161

Charbonneau, P., \& MacGregor, K. B. 1993, ApJ, 417, 762

Christensen-Dalsgaard, J., \& Schou, J. 1988, in Seismology of the Sun and Sun-like Stars, ed. E. J. Rolfe (ESA SP-286) (Noordwijk: ESA), 149

Christensen-Dalsgaard, J., Schou, J., \& Thompson, M. J. 1990, MNRAS, 242, 353

Cho, J. Y.-K., \& Polvani, L. M. 1996, Science, 273, 335

Clune, T. L., Elliott, J. R., Glatzmaier, G. A., Miesch, M., \& Toomre, J.

1998, Parallel Comp., submitted

\section{REFERENCES}

Corbard, T., Berthomieu, G., Morel, P., Provost, J., Schou, J., \& Tomczyk, S. 1997, A\&A, 324, 298

Corbard, T., Berthomieu, G., Provost, J., \& Morel, P. 1998, A\&A, 330, 1149

Durney, B. R. 1985, ApJ, 297, 787 1991, ApJ, 378, 378

Duvall, T. L. Jr. 1980, Sol. Phys., 66, 213

Duvall, T. L. Jr., Dziembowski, W. A., Goode, P. R., Gough, D. O., Harvey, J. W., \& Leibacher, J. W. 1984, Nature, 310, 22

Dziembowski, W. A., Goode, P. R., \& Libbrecht, K. G. 1989, ApJ, 337, L53 Edmonds, A. R. 1959, Angular Momentum in Quantum Mechanics (Princeton: Princeton Univ. Press)

Eff-Darwich, A. 1998, A\&AS, submitted

Eff-Darwich, A., \& Pérez-Hernández, F. 1997, A\&AS, 125, 391

Elliott, J. R. 1997, A\&A, 327, 1222

Elsworth, Y., Howe, R., Isaak, G. R., McLeod, C. P., Miller, B. A., New, R., Speake, C. C., \& Wheeler, S. J. 1994, ApJ, 434, 801

Elsworth, Y., Howe, R., Isaak, G. R., McLeod, C. P., Miller, B. A., New, R., Wheeler, S. J., \& Gough, D. O. 1995, Nature, 376, 669

Foukal, P. V. 1977, ApJ, 218, 539

Foukal, P. V., \& Jokipp, J. R. 1975, ApJ, 199, L71

Ghosal, S., \& Spiegel, E. A. 1991, Geophys. Astrophys. Fluid Dynam., 61, 161

Gilman, P. A. 1977, Geophys. Astrophys. Fluid Dynam., 8, 93 . 1979, ApJ, 231, 284

1983, ApJS, 53, 243 
Gilman, P. A. 1986, in Physics of the Sun, ed. P. A. Sturrock et al. (Dordrecht: Reidel), 1,95

Gilman, P. A., \& Foukal, P. V. 1979, ApJ, 229, 1179

Gilman, P. A., \& Miller, J. 1986, ApJS, 61, 585

Gilman, P. A., Morrow, C. A., \& DeLuca, E. E. 1989, ApJ, 338, 528

Glatzmaier, G. A. 1985, ApJ, 291, 300 1987, in The Internal Solar Angular Velocity, ed. B. R. Durney \& S. Sofia (Dordrecht: Reidel), 263

Glatzmaier, G. A., \& Gilman, P. A. 1982, ApJ, 256, 316

Glatzmaier, G. A., \& Toomre, J. 1995, in ASP Conf. Ser., 76, GONG'94: Helio- and Asteroseismology from Earth and Space, ed. R. K. Ulrich,

E. J. Rhodes, Jr., \& W. Däppen (San Francisco: ASP), 200

Goode, P. R., \& Dziembowski, W. A. 1991, Nature, 349, 223

Gough, D. O. 1977, in Proc. IAU Colloq. 36, The Energy Balance and Hydrodynamics of the Solar Chromosphere and Corona, ed. R. M. Bonnet \& P. Delache (Clermont-Ferrand: G. de Bussac), 3

. 1978, in EPS Workshop on Solar Rotation, ed. G. Belvedere \& L. Paternò (Catania: Catania Univ. Press), 337

. 1988, in Solar-Terrestrial Relationships and the Earth Environment in the Last Millennium, ed. G. Castagnoli-Cini (Bologna: Soc. Italiana di Fisica), 90 . 1997, Nature, 388, 324

Gough, D. O., Kosovichev, A. G., Sekii, T., Libbrecht, K. G., \& Woodard, M. F. 1993, in ASP Conf. Ser., 40, Inside the Stars, ed. W. W. Weiss \& A. Baglin (San Francisco: ASP), 93

Gough, D. O., \& McIntyre, M. E. 1998, Nature, in press

Gough, D. O., \& Stark, P. B. 1993a, in ASP Conf. Ser. 42, GONG 1992: Seismic Investigation of the Sun and Stars, ed. T. M. Brown (San Francisco: ASP), 221

. 1993b, ApJ, 415, 376

Gough, D. O., \& Toomre, J. 1991, ARA\&A, 29, 627

Hartmann, L. W., \& Noyes, R. W. 1987, ARA\&A, 25, 271

Harvey, J. W. 1995, Phys. Today, 48(10), 32

Harvey, J. W., et al. 1996, Science, 272, 1284

Hathaway, D. H. 1984, ApJ, 276, 316

Hathaway, D. H., et al. 1996, Science, 272, 1306

Hill, F., et al. 1996, Science, 272, 1292

Howard, R. F., \& LaBonte, B. J. 1980, ApJ, 239, L33

Howard, R. F., Gilman, P. A., \& Gilman, P. I. 1984, ApJ, 283, 373

Howe, R., \& Thompson, M. J. 1996, MNRAS, 281, 1385

. 1998a, in Proc. IAU Symp. 181, Sounding Solar and Stellar Interiors, ed. J. Provost \& F.-X. Schmider (Nice: Obs. Nice), 35 $1998 \mathrm{~b}, \mathrm{~A} \& \mathrm{~A}$, in press

Ingersoll, A. P. 1990, Science, 248, 308

Kosovichev, A. G. 1996, ApJ, 469, L61

Kosovichev, A. G., \& Schou, J. 1997, ApJ, 482, L207

Kosovichev, A. G., et al. 1997, Sol. Phys., 170, 43

1998, in preparation

Kumar, P., \& Quataert, E. J. 1997, ApJ, 475, L143

Larsen, R. M. 1997, in Proc. Interdisciplinary Inversion Workshop 5, ed.

B. H. Jacobsen (Aarhus: Aarhus Univ.), 123

Larsen, R. M., \& Hansen, P. C. 1997, A\&AS, 121, 587

Lazrek, M., et al. 1996, Sol. Phys., 166, 1

Libbrecht, K. G. 1989, ApJ, 336, 1092

McIntyre, M. E. 1994, in The Solar Engine and Its Influence on the Terrestrial Atmosphere and Climate, ed. E. Nesme-Ribes (Berlin: Springer), 293

Merryfield, W. J., Toomre, J., \& Gough, D. O. 1991, ApJ, 367, 658

Mestel, L., \& Weiss, N. O. 1987, MNRAS, 226, 123

Nesme-Ribes, E., Ferreira, E. N., \& Mein, P. 1993, A\&A, 274, 563

Pijpers, F. P. 1997, A\&A, 326, 1235

Pijpers, F. P., \& Thompson, M. J. 1992, A\&A, 262, L33

1994, A\&A, 281, 231

. 1996, MNRAS, 279, 498
Pulkkinen, P., Tuominen, I., Brandenburg, A., Nordlund, Å., \& Stein, R. F. 1993, A\&A, 267, 265

Rhodes, E. J., Cacciani, A., Korzennik, S. G., Tomczyk, S., Ulrich, R. K., \& Woodard, M. F. 1990, ApJ, 351, 687

Ritzwoller, M. H., \& Lavely, E. M. 1991, ApJ, 369, 557

Rosner, R. 1980, in Cool Stars, Stellar Systems, and the Sun, ed. A. K. Dupree (SAO SR-389) (Cambridge: SAO), 79

Rosner, R. \& Weiss, N. O. 1985, Nature, 317, 790

Rüdiger, G. 1980, Geophys. Astrophys. Fluid Dynam., 16, 239

. 1989, Differential Rotation and Steller Convection, Sun and SolarType Stars, The Fluid Mechanics of Astrophysics and Geophysics, vol. 5 (London: Gordon Breach)

Scherrer, P. H., et al. 1995, Sol. Phys., 62, 129

Schou, J. 1991a, in Lecture Notes in Physics 388, Challenges to Theories of the Structure of Moderate-Mass Stars, ed. D. Gough \& J. Toomre (Berlin: Springer), 81

. 1991b, in Lecture Notes in Physics 388, Challenges to Theories of the Structure of Moderate-Mass Stars, ed. D. Gough \& J. Toomre (Berlin: Springer), 93

. 1992, On the Analysis of Helioseismic Data, Ph.D. thesis, Aarhus Univ.

Schou, J., \& Brown, T. M. 1994, ApJ, 434, 378

Schou, J., et al. 1998, in Proc. IAU Symp. 185: New Eyes to See inside the Sun and Stars, ed. F.-L. Deubner, J. Christensen-Dalsgaard, \& D. W. Kurtz (Dordrecht: Kluwer), 141

Schou, J., Christensen-Dalsgaard, J., \& Thompson, M. J. 1994, ApJ, 433, 389

. 1995, in ASP Conf. Ser., 76, GONG'94: Helio- and Asteroseismology from Earth and Space, ed. R. K. Ulrich, E. J. Rhodes, Jr. \& W. Däppen (San Francisco: ASP), 528

Schou, J., Tomczyk, S. \& Thompson, M. J. 1996, Bull. Astron. Soc. India, 24,375

Sekii, T. 1993a, in ASP Conf. Ser. 42, GONG 1992: Seismic Investigation of the Sun and Stars, ed. T. M. Brown (San Francisco: ASP), 237 1993b, MNRAS, 264, 1018

1995, in Proc. Fourth SOHO Workshop Helioseismology, ed. J. T. Hoeksema et al. (ESA SP-376) (Noordwijk: ESA), 2, 285

Snodgrass, H. B. 1983, ApJ, 270, 288

Snodgrass, H. B., Howard, R. F., \& Webster, L. 1985, Sol. Phys., 95, 221

Solanki, S. K. 1993, Space Sci. Rev, 63, 1

Spiegel, E. A., \& Weiss, N. O. 1980, Nature, 287, 616

Spiegel, E. A., \& Zahn, J.-P. 1992, A\&A, 265, 106

Spruit, H. C. 1987, in The Internal Solar Angular Velocity, ed. B. R. Durney \& S. Sofia (Dordrecht: Reidel), 185

Thompson, M. J. 1993, in ASP Conf. Ser. 42, GONG 1992: Seismic Investigation of the Sun and Stars, ed. T. M. Brown (San Francisco: ASP), 141 Thompson, M. J., et al. 1996, Science, 272, 1300

Tomczyk, S., Schou, J., \& Thompson, M. J. 1995a, ApJ, 448, L57

Tomczyk, S., Streander, K., Card, G., Elmore, D., Hull, H., \& Cacciani, A., 1995b, Sol. Phys., 159, 1

Toomre, J., \& Brummell, N. H. 1995, in Proc. Fourth SOHO Workshop Helioseismology, ed. J. T. Hoeksema et al. (ESA SP-376) (Noordwijk: ESA), 1, 47

Ulrich, R. K., Boyden, J. E., Webster, L., Snodgrass, H. B., Padilla, S. P., Gilman, P. A., \& Shieber, T. 1988, Sol. Phys., 117, 291

Weiss, N. O. 1994, in Lectures on Solar and Planetary Dynamos, ed. M. R. E. Proctor \& A. D. Gilbert (Cambridge: Cambridge Univ. Press), 59

Wilcox, J. M., \& Howard, R. F. 1970, Sol. Phys., 12, 23

Wilson, P. R. 1994, Solar Activity Cycles (Cambridge: Cambridge Univ. Press)

Wilson, P. R., \& Burtonclay, D. 1995, ApJ, 438, 445

Wilson, P. R., Burtonclay, D., \& Li, Y. 1996, ApJ, 470, 621

Zahn, J.-P., Talon, S., \& Matias, J. 1997, A\&A, 322, 320 